\title{
Complex Singularities of the Burgers System and Renormalization Group Method
}

\author{
Dong Li and Ya. G. Sinai
}

\begin{abstract}
We consider complex-valued solutions of the $n$-dimensional Burgers' system, $n>1$. We show that there exists an open set in the space of $n^{2}+5 n-2 / 2$-parameter families of initial conditions such that for each family from this set there are values of parameters for which the solution develops blow up in finite time.
\end{abstract}

\section{Introduction}

The n-dimensional Burgers system is a modification of the n-dimensional Navier-Stokes system. It is written for an unknown n-dimensional vector $u(x, t)=\left(u_{1}(x, t), \ldots, u_{n}(x, t)\right)$ and in the absence of the external forcing has the form

$$
\frac{\partial u_{j}}{\partial t}+\sum_{k=1}^{n} \frac{\partial u_{j}}{\partial x_{k}} u_{k}=\Delta u_{j}, 1 \leq j \leq n .
$$

The viscosity is taken to be $1, x \in R^{n}$.

(1) has an invariant submanifold of gradient-like solutions, i.e., solutions which are gradients of some functions. The analysis of these solutions is done with the help of the so-called Hopf-Cole transformation (see $[\mathbf{H C}]$ ). They are much simpler than general solutions of the Burgers system.

Concerning general solutions there is a theorem by Ladyzhenskaya (see $[\mathbf{L a}]$ ) which gives the existence and uniqueness of solutions in Sobolev spaces. However, this result remains a folklore theorem because $[\mathbf{L a}]$ dos not contain a detailed proof.

In this paper we consider complex solutions of (1) and show that they can develop singularities in finite time. For gradient-like solutions this was proven recently by Poláčik and Šverák (see $[\mathbf{P S}]$ ). The result and the method of the present paper are similar to the ones in our joint 
paper $[\mathbf{L S}]$ where we proved the same statement for the 3-dimensional Navier-Stokes system. As in $[\mathbf{L S}]$ write the Fourier transform of $u$ in the form $-i v(k, t)$. Then for $v$ we have the following equation:

$$
v(k, t)=e^{-t|k|^{2}} v(k, 0)+\int_{0}^{t} e^{-(t-s)|k|^{2}} d s \int_{R^{n}}\left\langle v\left(k-k^{\prime}, s\right), k^{\prime}\right\rangle v\left(k^{\prime}, s\right) d k^{\prime} .
$$

In this paper we consider real solutions of (2). Real solutions of (1) appear from odd functions $v(k, t)$.

As in $[\mathbf{L S}]$ we use power series which represent solutions of (2). Let $v_{A}(k, 0)=A v(k, 0)$. Then the solution $v_{A}(k, t)$ of $(2)$ can be written in the form

$$
v_{A}(k, t)=e^{-t|k|^{2}} A v(k, 0)+\int_{0}^{t} e^{-(t-s)|k|^{2}} \sum_{p>1} A^{p} g_{p}(k, s) d s
$$

The substitution of (3) into (2) gives the system of recurrent relations for the functions $g_{p}$ :

$$
g_{1}(k, s)=\exp \left\{-s|k|^{2}\right\} v(k, 0),
$$

$$
g_{2}(k, s)=\int_{R^{n}}\left\langle v\left(k-k^{\prime}, 0\right), k^{\prime}\right\rangle v\left(k^{\prime}, 0\right) \cdot \exp \left\{-s\left|k-k^{\prime}\right|^{2}-s\left|k^{\prime}\right|^{2}\right\} d^{n} k^{\prime},
$$

$$
\begin{aligned}
g_{p}(k, s)= & \int_{0}^{s} d s_{2} \int_{R^{n}}\left\langle v\left(k-k^{\prime}, 0\right), k^{\prime}\right\rangle g_{p-1}\left(k^{\prime}, s_{2}\right) \cdot \\
& \cdot \exp \left\{-s\left|k-k^{\prime}\right|^{2}-\left(s-s_{2}\right)\left|k^{\prime}\right|^{2}\right\} d^{n} k^{\prime} \\
+ & \sum_{\substack{p_{1}+p_{2}=p \\
p_{1}, p_{2}>1}} \int_{0}^{s} d s_{1} \int_{0}^{s} d s_{2} \int_{R^{n}}\left\langle g_{p_{1}}\left(k-k^{\prime}, s_{1}\right), k^{\prime}\right\rangle \cdot \\
& \cdot g_{p_{2}}\left(k^{\prime}, s_{2}\right) \cdot \exp \left\{-\left(s-s_{1}\right)\left|k-k^{\prime}\right|^{2}-\left(s-s_{2}\right)\left|k^{\prime}\right|^{2}\right\} d^{n} k^{\prime} \\
+ & \int_{0}^{s} d s_{1} \int\left\langle g_{p-1}\left(k-k^{\prime}, s_{1}\right), k^{\prime}\right\rangle v\left(k^{\prime}, 0\right) \cdot \\
& \cdot \exp \left\{-\left(s-s_{1}\right)\left|k-k^{\prime}\right|^{2}-s\left|k^{\prime}\right|^{2}\right\} d^{n} k^{\prime} .
\end{aligned}
$$

The same methods as in [Si 2] allow to prove that the series (3) is converging for small $t$. We can see from (6) that the first and the last terms are different from the other terms in (6) because they contain explicitly the initial condition.

As in $[\mathbf{L S}]$ we modify (6) by extracting the main part of (6). In this way we come to a nonlinear equation giving the "fixed point" of the 
renormalization group (see $\S 2$ ). In the case of Burgers system solutions to this equation are simpler than in $[\mathbf{L S}]$ and consist only of Gaussian functions. In $\S 3$ following the same line as in $[\mathbf{L S}]$ we study the spectrum of the corresponding linearized group. Then using the same strategy as in $[\mathbf{L S}]$ we prove our main result whose formulation is given below.

Main theorem. Let $\tau_{n}=\frac{n^{2}+5 n-2}{2}$. There exists an open set in the space of $\tau_{n}$-parameter families of initial conditions such that for each family from this set one can find the values of parameters so that the solution having the corresponding initial condition develops a blow up at time $t_{c r}$. If $E(t)=\int_{R^{n}}\langle v(k, t), v(k, t)\rangle d k, \Omega(t)=$ $\int_{R^{n}}\langle k, k\rangle\langle v(k, t), v(k, t)\rangle d k$ are the energy and the enstrophy of the solution, then $E(t) \approx \frac{1}{\left(t_{c r}-t\right)^{5}}, \Omega(t) \approx \frac{1}{\left(t_{c r}-t\right)^{7}}$ as $t \rightarrow t_{c r}$.

The first author is supported by the NSF Grant DMS 0111298. The second author is supported by NSF Grant DMS 0600996.

\section{The Derivation of the Equation for the Fixed Point and the Analysis of Its Solutions}

Take some number $k^{(0)}$ which later will be assumed to be sufficiently large and introduce the vector $\mathcal{K}^{(r)}=r k^{(0)}(1,1, \ldots, 1)$. These will be the points near which all $g_{r}$ will be concentrated,We write $k=\mathcal{K}^{(r)}+$ $\sqrt{r k^{(0)}} \cdot Y, Y \in R^{n}$. Thus instead of $k$ we have the new variable $Y=$ $\left(Y_{1}, \ldots, Y_{n}\right)$ which typically will take values $O(1)$. Put $\kappa^{(0,0)}=(1, \ldots, 1)$ and $\kappa^{(0)}=\left(k^{(0)}, \ldots, k^{(0)}\right)$.

In all integrals over $s_{1}, s_{2}$ in (6) make another change of variables $s_{j}=s\left(1-\frac{\theta_{j}}{p_{j}^{2}}\right), j=1,2$. Instead of the variable of integration $k^{\prime}$ introduce $Y^{\prime}$ where $k^{\prime}=\mathcal{K}^{\left(p_{2}\right)}+\sqrt{p k^{(0)}} Y^{\prime}$. All this is very much similar to $[\mathbf{L S}]$. Write $\tilde{g}_{r}(Y, s)=g_{r}\left(\mathcal{K}^{(r)}+\sqrt{r k^{(0)}} Y, s\right), \gamma=\frac{p_{1}}{p}, \frac{p_{2}}{p}=1-\gamma$. Then (6) gives us a slightly modified recurrent equation:

$$
\begin{aligned}
& \tilde{g}_{p}(Y, s) \\
& =g_{p}\left(\mathcal{K}^{(p)}+\sqrt{p k^{(0)}} Y, s\right) \\
& =\left(p k^{(0)}\right)^{\frac{n+2}{2}}\left[\sum_{\substack{p_{1}, p_{2} \geq 1 \\
p_{1}+p_{2}=p}} \int_{0}^{p_{1}^{2}} d \theta_{1} \int_{0}^{p_{2}^{2}} d \theta_{2} \cdot \frac{1}{p_{1}^{2} \cdot p_{2}^{2}} \cdot\right. \\
& \cdot \int_{R^{n}}\left\langle\tilde{g}_{p_{1}}\left(\frac{Y-Y^{\prime}}{\sqrt{\gamma}},\left(1-\frac{\theta_{1}}{p_{1}^{2}}\right) s\right), \frac{p_{2}}{p} \kappa^{(0,0)}+\frac{Y^{\prime}}{\sqrt{p k^{(0)}}}\right\rangle .
\end{aligned}
$$




$$
\begin{aligned}
& \cdot \tilde{g}_{p_{2}}\left(\frac{Y^{\prime}}{\sqrt{1-\gamma}},\left(1-\frac{\theta_{2}}{p_{2}^{2}}\right) s\right) \cdot \\
& \left.\cdot \exp \left\{-\theta_{1}\left|\kappa^{(0)}+\sqrt{k^{(0)}} \frac{Y-Y^{\prime}}{\sqrt{p} \cdot \gamma}\right|^{2}-\theta_{2}\left|\kappa^{(0)}+\frac{\sqrt{k^{(0)}} Y^{\prime}}{\sqrt{p}(1-\gamma)}\right|^{2}\right\} d^{n} Y^{\prime}\right] .
\end{aligned}
$$

This is our main recurrent relation. It is of some importance that in front of (7) we have the factor $p^{\frac{n+2}{2}}$ and inside the sum the factor $\frac{1}{p_{1}^{2}} \cdot \frac{1}{p_{2}^{2}}$.

As $p \rightarrow \infty$ the recurrent equation (7) takes some limiting form. The main contribution to (7) comes from $p_{1}, p_{2}$ of order $p$. If $Y, Y^{\prime}=O(1)$ then $\frac{Y-Y^{\prime}}{\sqrt{p}}, \frac{Y^{\prime}}{\sqrt{p}}$ are small compared to $\kappa^{(0)}=\left(k^{(0)}, \ldots, k^{(0)}\right)$. Therefore in the main order of magnitude the Gaussian term in (7) can be replaced by $\exp \left\{-\left(\theta_{1}+\theta_{2}\right)\left|k^{(0)}\right|^{2}\right\}, s_{1}$ and $s_{2}$ can be replaced by $s$ and the integrations over $\theta_{1}, \theta_{2}$ and $Y^{\prime}$ can be done separately. Thus instead of (7) we get a simpler recurrent relation:

$$
\begin{aligned}
& \tilde{g}_{p}(Y, s)=\left(k^{(0)}\right)^{\frac{n-6}{2}} p^{\frac{n+2}{2}} \sum_{\substack{p_{1}, p_{2}>p^{1 / 2} \\
p_{1}+p_{2}=p}} \frac{1}{p_{1}^{2} \cdot p_{2}^{2}} . \\
& \cdot \int_{R^{n}}\left\langle\tilde{g}_{p_{1}}\left(\frac{Y-Y^{\prime}}{\sqrt{\gamma}}, s\right), \frac{p_{2}}{p} \kappa^{(0,0)}+\frac{Y^{\prime}}{\sqrt{p k^{(0)}}}\right\rangle . \\
& \cdot \tilde{g}_{p_{2}}\left(\frac{Y^{\prime}}{\sqrt{1-\gamma}}, s\right) d^{n} Y^{\prime} .
\end{aligned}
$$

In our approximation the inner product in (8) can be replaced by

$$
\left\langle\tilde{g}_{p_{1}}\left(\frac{Y-Y^{\prime}}{\sqrt{\gamma}}, s\right), \frac{p_{2}}{p} \kappa^{(0,0)}\right\rangle=\frac{p_{2}}{p} \sum_{j=1}^{n} \tilde{g}_{p_{1}}^{(j)}\left(\frac{Y-Y^{\prime}}{\sqrt{\gamma}}, s\right),
$$

where $\tilde{g}_{p_{1}}=\left(\tilde{g}_{p_{1}}^{(1)}, \ldots, \tilde{g}_{p_{1}}^{(n)}\right)$.

We come to even simpler recurrent relation instead of (8):

$$
\begin{aligned}
\tilde{g}_{p}(Y, s)=\left(k^{(0)}\right)^{\frac{n-6}{2}} p^{\frac{n+2}{2}} \sum_{\substack{p_{1}, p_{2}>p^{1 / 2} \\
p_{1}+p_{2}=p}} \frac{1}{p_{1}^{2} \cdot p_{2}^{2}} \\
\\
\quad \int_{R^{n}} \frac{p_{2}}{p} \sum_{j=1}^{n} \tilde{g}_{p_{1}}^{(j)}\left(\frac{Y-Y^{\prime}}{\sqrt{\gamma}}, s\right) \cdot \tilde{g}_{p_{2}}\left(\frac{Y^{\prime}}{\sqrt{1-\gamma}}, s\right) d^{n} Y^{\prime} .
\end{aligned}
$$

As in $[\mathbf{L S}]$ we make the following inductive assumption concerning the form of $\tilde{g}_{p}(Y, s)$ : there exist intervals $S^{(p)}=\left[S_{-}^{(p)}, S_{+}^{(p)}\right], S^{(p+1)} \subset$ $S^{(p)}$, on the time axis, the functions $Z(s), \Lambda(s)$ defined for $s \in S^{(1)}$, 
positive numbers $\sigma^{(j)}, 1 \leq j \leq n$, such that for all $r<p$

$$
\begin{gathered}
\tilde{g}_{r}(Y, s)=Z(s) \Lambda_{p}^{r}(s) r^{\frac{4-n}{2}} \prod_{j=1}^{n} \sqrt{\frac{\sigma^{(j)}}{2 \pi}} \exp \left(-\frac{\sigma^{(j)}}{2}\left|Y_{j}\right|^{2}\right) . \\
\cdot\left(H(Y)+\delta^{(r)}(Y, s)\right) .
\end{gathered}
$$

We derive below the equation for the function $H$. The main part of the proof is to organize the inductive process in such a way that the remainders $\delta^{(r)}$ tend to zero as $r \rightarrow \infty$. The substitution of (10) into (9) gives

$$
\begin{aligned}
& \tilde{g}_{p}(Y, s)=\left(k^{(0)}\right)^{\frac{n-6}{2}} Z(s)^{2} \cdot p^{\frac{4-n}{2}} \Lambda^{p}(s) \sum_{\gamma=\frac{p_{1}}{p}} \frac{1}{p} \cdot(1-\gamma) \\
& \cdot \int_{R^{n}}\left(\sum_{j=1}^{n} H^{(j)}\left(\frac{Y-Y^{\prime}}{\sqrt{\gamma}}\right)\right) H\left(\frac{Y^{\prime}}{\sqrt{1-\gamma}}\right) . \\
& \cdot \prod_{j=1}^{n} \sqrt{\frac{\sigma^{(j)}}{2 \pi \gamma} \exp \left(-\frac{\sigma^{(j)}}{2 \gamma}\left|Y_{j}-Y_{j}^{\prime}\right|^{2}\right) \cdot} \\
& \cdot \prod_{j=1}^{n} \sqrt{\frac{\sigma^{(j)}}{2 \pi(1-\gamma)}} \exp \left(-\frac{\sigma^{(j)}}{2(1-\gamma)}\left|Y_{j}^{\prime}\right|^{2}\right) d^{n} Y^{\prime} .
\end{aligned}
$$

Here $H=\left(H^{(1)}, \ldots, H^{(n)}\right)$ and we do not mention explicitly the dependence of $H$ on $s$. The last sum looks like a Riemannian integral sum and as $p \rightarrow \infty$ its limit takes the form:

$$
\begin{aligned}
& H(Y) \prod_{j=1}^{n} \sqrt{\frac{\sigma^{(j)}}{2 \pi}} \exp \left(-\frac{\sigma^{(j)}}{2}\left|Y_{j}\right|^{2}\right) \\
&=\left(k^{(0)}\right)^{\frac{n-6}{2}} Z(s) \cdot \int_{0}^{1} d \gamma(1-\gamma) \cdot \\
& \cdot \int_{R^{n}}\left(\sum_{j=1}^{n} H^{(j)}\left(\frac{Y-Y^{\prime}}{\sqrt{\gamma}}\right)\right) H\left(\frac{Y^{\prime}}{\sqrt{1-\gamma}}\right) \cdot \\
& \cdot \prod_{j=1}^{n} \sqrt{\frac{\sigma^{(j)}}{2 \pi \gamma}} \exp \left(-\frac{\sigma^{(j)}}{2 \gamma}\left|Y_{j}-Y_{j}^{\prime}\right|^{2}\right) \cdot \\
& \cdot \prod_{j=1}^{n} \sqrt{\frac{\sigma^{(j)}}{2 \pi(1-\gamma)}} \exp \left(-\frac{\sigma^{(j)}}{2(1-\gamma)}\left|Y_{j}^{\prime}\right|^{2}\right) d^{n} Y^{\prime}
\end{aligned}
$$


Put $Z(s)=\left(k^{(0)}\right)^{\frac{6-n}{2}}$. The final equation does not contain $k^{(0)}$ and we have

$$
\begin{aligned}
& H(Y) \prod_{j=1}^{n} \sqrt{\frac{\sigma^{(j)}}{2 \pi}} \exp \left(-\frac{\sigma^{(j)}}{2}\left|Y_{j}\right|^{2}\right) \\
& =\int_{0}^{1} d \gamma(1-\gamma) \int_{R^{n}}\left(\sum_{j=1}^{n} H^{(j)}\left(\frac{Y-Y^{\prime}}{\sqrt{\gamma}}\right)\right) H\left(\frac{Y^{\prime}}{\sqrt{1-\gamma}}\right) \\
& \cdot \prod_{j=1}^{n} \sqrt{\frac{\sigma^{(j)}}{2 \pi \gamma}} \exp \left(-\frac{\sigma^{(j)}}{2 \gamma}\left|Y_{j}-Y_{j}^{\prime}\right|^{2}\right) \cdot \\
& \cdot \prod_{j=1}^{n} \sqrt{\frac{\sigma^{(j)}}{2 \pi(1-\gamma)}} \exp \left(-\frac{\sigma^{(j)}}{2(1-\gamma)}\left|Y_{j}^{\prime}\right|^{2}\right) d^{n} Y^{\prime}
\end{aligned}
$$

This equation is the equation for the fixed point of our renormalization group. It is analogous to the fixed point equation from $[\mathbf{L S}]$.

The solutions of (11) have natural scaling with respect to the parameters $\sigma^{(j)}, 1 \leq j \leq n$. Namely, if we solve the equation (11) for $\sigma^{(j)}=1,1 \leq j \leq n$ and denote the corresponding solution by $H(Y)$, then the general solution for arbitrary $\sigma=\left(\sigma^{(1)}, \ldots, \sigma^{(n)}\right)$ is given by the formula

$$
H_{(\sigma)}(Y)=\left(\prod_{j=1}^{n} \sqrt{\sigma^{(j)}}\right) H\left(\sqrt{\sigma^{(1)}} Y_{1}, \ldots, \sqrt{\sigma^{(n)}} Y_{n}\right)
$$

Similar scaling was mentioned in the case of Navier-Stokes system (see $[\mathbf{L S}]$ ). Thus, it is enough to consider (11) with $\sigma^{(j)}=1,1 \leq j \leq n$.

As in $[\mathbf{L S}]$ we use expansions over Hermite polynomials:

$$
\begin{aligned}
& H^{(j)}\left(Y_{1}, \ldots, Y_{n}\right) \\
& =\sum_{m_{1}, \ldots, m_{n} \geq 0} h_{m_{1}, \ldots, m_{n}}^{(j)} H e_{m_{1}}\left(Y_{1}\right) \cdots H e_{m_{n}}\left(Y_{n}\right), \quad j=1, \ldots, n
\end{aligned}
$$

where $H e_{m}(z)$ are the Hermite polynomials of degree $m$ with respect to the Gaussian density $\frac{1}{\sqrt{2 \pi}} \exp \left\{-\frac{1}{2} z^{2}\right\}$. Recall the following properties of Hermite polynomials:

$$
\begin{aligned}
& \text { 1. } H e_{0}(z)=1, \quad z H e_{0}(z)=z=H e_{1}(z) . \\
& \text { 2. } z H e_{m}(z)=H e_{m+1}(z)+m H e_{m-1}(z), m>0
\end{aligned}
$$




$$
\begin{aligned}
& \text { 3. } \int_{\mathbb{R}^{1}} H e_{m_{1}}\left(\frac{Y-Y^{\prime}}{\sqrt{\gamma}}\right) \frac{1}{\sqrt{2 \pi}} \exp \left\{-\frac{\left|Y-Y^{\prime}\right|^{2}}{2 \gamma}\right\} . \\
& \cdot H e_{m_{2}}\left(\frac{Y^{\prime}}{\sqrt{1-\gamma}}\right) \frac{1}{\sqrt{2 \pi}} \exp \left\{-\frac{\left|Y^{\prime}\right|^{2}}{2(1-\gamma)}\right\} d Y^{\prime} \\
& =\gamma^{\frac{m_{1}+1}{2}}(1-\gamma)^{\frac{m_{2}+1}{2}} H e_{m_{1}+m_{2}}(Y) \frac{1}{\sqrt{2 \pi}} \exp \left\{-\frac{|Y|^{2}}{2}\right\} .
\end{aligned}
$$

Substituting (13) into (11) and using 1),2),3), we come to the system of equations for the coefficients $h_{m_{1}, \ldots, m_{n}}^{(j)}$ which is equivalent to (11):

$$
\begin{aligned}
& h_{m_{1}, \ldots, m_{n}}^{(j)} \\
& =\sum_{\substack{m_{1}^{\prime}+m_{1}^{\prime \prime}=m_{1} \\
\vdots \\
m_{n}^{\prime}+m_{n}^{\prime \prime}=m_{n}}}\left(\int_{0}^{1} \gamma^{\frac{m^{\prime}}{2}}(1-\gamma)^{\frac{m^{\prime \prime}+2}{2}} d \gamma\right) \cdot \sum_{k=1}^{n} h_{m_{1}^{\prime}, \ldots, m_{n}^{\prime}}^{(k)} h_{m_{1}^{\prime \prime}, \ldots, m_{n}^{\prime \prime}}^{(j)}
\end{aligned}
$$

where $m^{\prime}=m_{1}^{\prime}+\cdots+m_{n}^{\prime}, m^{\prime \prime}=m_{1}^{\prime \prime}+\cdots+m_{n}^{\prime \prime}$.

For the coefficient $h^{(j)}\left(m_{1}, \ldots, m_{n}\right)$ we define $d=m_{1}+\cdots+m_{n}$. We solve (14) for different values of $d$.

Case 1: $d=0$. In this case we have

$$
h^{(j)}(0, \ldots, 0)=\frac{1}{2}\left(\sum_{k=1}^{n} h^{(k)}(0, \ldots, 0)\right) h^{(j)}(0, \ldots, 0)
$$

We are interested in solutions for which all $h^{(j)}(0, \ldots, 0)$ are nonzero. Then we must have

$$
\sum_{k=1}^{n} h^{(k)}(0, \ldots, 0)=2 .
$$

Case 2: $d=1$. Here it is not difficult to show that the only possible solution is $h^{(k)}\left(m_{1}, \ldots, m_{n}\right)=0$ for any $m_{1}+\cdots+m_{n}=1$ and $1 \leq k \leq$ $n$.

Case 3: $d>1$. A simple induction on $d$ shows that all terms with the given $d$ are zero.

From all these three cases it follows easily that any solution of (14) is given by $\left(h^{(1)}(0, \ldots, 0), \cdots, h^{(n)}(0, \ldots, 0)\right)$ satisfying $(15)$ while all other terms are zero. We formulate the final result as the following theorem

TheOREM 2.1. Let $\sigma^{(1)}>0, \ldots, \sigma^{(n)}>0$ and $x^{(j)}=h^{(j)}(0, \ldots, 0)$, $1 \leq j \leq n-1$ are arbitrary and $x^{(n)}=2-\sum_{k=1}^{n-1} x^{(k)}$. Then there exists 
a solution of (11) having the following form

$$
G^{\sigma, x}(Y)=\left(\prod_{j=1}^{n} \sqrt{\sigma^{(j)}}\right)\left\{\prod_{j=1}^{n} \sqrt{\frac{\sigma^{(j)}}{2 \pi}} \exp \left(-\frac{\sigma^{(j)}\left|Y_{j}\right|^{2}}{2}\right)\right\} \cdot x
$$

where $\sigma=\left(\sigma^{(1)}, \ldots, \sigma^{(n)}\right), x=\left(x^{(1)}, \ldots, x^{(n)}\right)$. In other words all fixed points of our renormalization group are Gaussian!

\section{The Linearization Near Fixed Point}

As in $[\mathbf{L S}]$ the strategy of the proof of the main result is based on the method of renormalization group. Let us write

$\tilde{g}_{r}(Y, s) \Lambda^{-r}(s) Z^{-1}(s) \cdot r^{\frac{n-4}{2}} \prod_{j=1}^{n} \frac{\sqrt{2 \pi}}{\sigma^{(j)}} \exp \left(\frac{\sigma^{(j)}}{2}\left|Y_{j}\right|^{2}\right)=x+\delta^{(r)}(\gamma, Y, s)$

where $\delta^{(r)}(\gamma, Y, s)=\left\{\delta_{j}^{(r)}(\gamma, Y, s), 1 \leq j \leq n\right\}=\tilde{\delta}^{(p)}(\gamma, Y, s), \gamma=\frac{r}{p}$ and $\gamma \leq 1$. It is natural to consider the set of functions $\left\{\tilde{\delta}^{(p)}(\gamma, Y, s)\right\}$ as a small perturbation of our fixed point. When we go from $p$ to $p+1$

$$
\tilde{\delta}^{(p+1)}(\gamma, Y, s)=\tilde{\delta}^{(p)}\left(\frac{p+1}{p} \gamma, Y, s\right), \quad \gamma \leq \frac{p}{p+1},
$$

The formula for $\tilde{\delta}^{(p+1)}(1, Y, s)$ follows from (11):

$$
\begin{aligned}
& \tilde{\delta}_{k}^{(p)}(1, Y, s) \prod_{j=1}^{n} \sqrt{\frac{\sigma^{(j)}}{2 \pi}} \exp \left(-\frac{\sigma^{(j)}}{2}\left|Y_{j}\right|^{2}\right) \\
& =\int_{0}^{1} d \gamma(1-\gamma) \int_{R^{n}}\left(\sum_{j=1}^{n} \tilde{\delta}_{j}^{(p)}\left(\gamma, \frac{Y-Y^{\prime}}{\sqrt{\gamma}}, s\right) H^{(k)}\left(\frac{Y^{\prime}}{\sqrt{1-\gamma}}\right)\right. \\
& \left.\quad+\sum_{j=1}^{n} H^{(j)}\left(\frac{Y-Y^{\prime}}{\sqrt{\gamma}}\right) \tilde{\delta}_{k}^{(p)}\left(1-\gamma, \frac{Y^{\prime}}{\sqrt{1-\gamma}}, s\right)\right) . \\
& \cdot \prod_{j=1}^{n} \sqrt{\frac{\sigma^{(j)}}{2 \pi \gamma}} \exp \left(-\frac{\sigma^{(j)}}{2 \gamma}\left|Y_{j}-Y_{j}^{\prime}\right|^{2}\right) \cdot \\
& \quad \prod_{j=1}^{n} \sqrt{\frac{\sigma^{(j)}}{2 \pi(1-\gamma)}} \exp \left(-\frac{\sigma^{(j)}}{2(1-\gamma)}\left|Y_{j}^{\prime}\right|^{2}\right) d^{n} Y^{\prime},
\end{aligned}
$$

where $\tilde{\delta}_{k}^{(p)}$ is the $k$-th component of $\tilde{\delta}^{(p)}$. We did not include in the last expression terms which are quadratic in $\tilde{\delta}^{(p+1)}$ because in this section we consider only the linearized part. 
Definition 3.1. A function $\Phi_{\alpha}(Y), Y=\left(Y_{1}, \ldots, Y_{n}\right)$ with values in $R^{n}$ is called an eigen-function if the function $\Phi_{\alpha}(\gamma, Y)=\gamma^{\alpha} \cdot \Phi_{\alpha}(Y)$ satisfies the equation:

$$
\begin{aligned}
\tilde{\Phi}_{\alpha, k}(Y) \prod_{j=1}^{n} \sqrt{\frac{\sigma^{(j)}}{2 \pi}} \exp \left(-\frac{\sigma^{(j)}}{2}\left|Y_{j}\right|^{2}\right) \\
=\int_{0}^{1} d \gamma(1-\gamma) \int_{R^{n}}\left(\sum_{j=1}^{n} \gamma^{\alpha} \Phi_{\alpha, j}\left(\frac{Y-Y^{\prime}}{\sqrt{\gamma}}\right) H^{(k)}\left(\frac{Y^{\prime}}{\sqrt{1-\gamma}}\right)\right. \\
\left.\quad+\sum_{j=1}^{n} H^{(j)}\left(\frac{Y-Y^{\prime}}{\sqrt{\gamma}}\right)(1-\gamma)^{\alpha} \Phi_{\alpha, k}\left(\frac{Y^{\prime}}{\sqrt{1-\gamma}}\right)\right) \cdot \\
\cdot \prod_{j=1}^{n} \sqrt{\frac{\sigma^{(j)}}{2 \pi \gamma}} \exp \left(-\frac{\sigma^{(j)}}{2 \gamma}\left|Y_{j}-Y_{j}^{\prime}\right|^{2}\right) \cdot \\
\quad \cdot \prod_{j=1}^{n} \sqrt{\frac{\sigma^{(j)}}{2 \pi(1-\gamma)}} \exp \left(-\frac{\sigma^{(j)}}{2(1-\gamma)}\left|Y_{j}^{\prime}\right|^{2}\right) d^{n} Y^{\prime} .
\end{aligned}
$$

In the last expression $\Phi_{\alpha, j}(\gamma, Y)$ is the $j$-th component of $\Phi_{\alpha}(Y)$.

The eigen-function corresponding to $\alpha$ will be denoted by $\Phi_{\alpha}$, its components will be denoted by $\Phi_{\alpha, j}$.

The meaning of the definition 3.1 is the following. Assume that we have a perturbation proportional to $\delta^{(r)}(Y)=\left(\frac{r}{p}\right)^{\alpha} \Phi_{\alpha}(Y), r<p$. If we apply (17) then in the main order of magnitude we shall get $\Phi_{\alpha}(Y)$.

Below we study in detail the set of eigen-functions $\Phi_{\alpha}$. If $\alpha>0$, $\alpha=0, \alpha<0$ then the corresponding eigen-functions are called unstable, neutral or stable correspondingly. We shall show that there exist the eigen-value $\alpha=1$ of multiplicity $\nu_{1}=1$, the eigen-value $\alpha=\frac{1}{2}$ of multiplicity $\nu_{1 / 2}=n-1$ and the eigen-value $\alpha=0$ of multiplicity $\nu_{0}=\frac{n^{2}+3 n-2}{2}$. All other eigen-values are stable. In view of the above mentioned scaling invariance it is enough to consider $\sigma^{(1)}=\cdots=\sigma^{(n)}=$ 1. We use again the expansion over Hermite polynomials:

$$
\begin{aligned}
& \Phi_{\alpha, 1, \ldots, 1}^{(j)}(Y) \\
& =\Phi_{\alpha}^{(j)}(Y) \\
& =\sum_{m_{1}, \ldots, m_{n}} f_{\alpha, j}\left(m_{1}, \ldots, m_{n}\right) H e_{m_{1}}\left(Y_{1}\right) \cdots H e_{m_{n}}\left(Y_{n}\right), 1 \leq j \leq n .
\end{aligned}
$$


Then we come to the linear system of recurrent relations

$$
\begin{aligned}
& f_{\alpha, j}\left(m_{1}, \ldots, m_{n}\right) \\
& =\sum_{m_{1}^{\prime}+m_{1}^{\prime \prime}=m_{1}}\left(\int_{0}^{1} \gamma^{\frac{m^{\prime}}{2}}(1-\gamma)^{\frac{m^{\prime \prime}+2}{2}+\alpha} d \gamma\right) \cdot \sum_{k=1}^{n} h_{m_{1}^{\prime}, \ldots, m_{n}^{\prime}}^{(k)} f_{\alpha, j}\left(m_{1}^{\prime \prime}, \ldots, m_{n}^{\prime \prime}\right) \\
& \quad \vdots \vdots \\
& m_{n}^{\prime}+m_{n}^{\prime \prime}=m_{n} \\
& \quad+\left(\int_{0}^{1} \gamma^{\frac{m^{\prime}}{2}+\alpha}(1-\gamma)^{\frac{m^{\prime \prime}+2}{2}} d \gamma\right) \cdot \sum_{k=1}^{n} f_{\alpha, k}\left(m_{1}^{\prime}, \ldots, m_{n}^{\prime}\right) h_{m_{1}^{\prime \prime}, \ldots, m_{n}^{\prime \prime}}^{(j)}
\end{aligned}
$$

where $h_{m_{1}, \ldots, m_{n}}^{(j)}$ are coefficients of the expansion of $H(Y)$. In our case $h^{(j)}\left(m_{1}, \ldots, m_{n}\right)=0$ if $m_{1}+\cdots+m_{n}>0$ and $\sum_{k=1}^{n} h^{(k)}(0, \ldots, 0)=2$. Denote $h^{(k)}(0, \ldots, 0)=x^{(k)}$. Then we get

$$
\begin{aligned}
f_{\alpha, j}\left(m_{1}, \ldots, m_{n}\right) & =\left(2 \int_{0}^{1} \gamma^{\frac{m}{2}+1+\alpha} d \gamma\right) f_{\alpha, j}\left(m_{1}, \ldots, m_{n}\right) \\
& +\left(\int_{0}^{1} \gamma^{\frac{m}{2}+\alpha}(1-\gamma) d \gamma\right)\left(\sum_{k=1}^{n} f_{\alpha, k}\left(m_{1}, \ldots, m_{n}\right)\right) x^{(j)} .
\end{aligned}
$$

After summation over $j, 1 \leq j \leq n$, and using the fact that $\sum_{j=1}^{n} x^{(j)}=$ 2 we come to the equation

$$
\left(\sum_{k=1}^{n} f_{\alpha, k}\left(m_{1}, \ldots, m_{n}\right)\right) \cdot\left(1-2 \int_{0}^{1} \gamma^{\frac{m}{2}+\alpha} d \gamma\right)=0 .
$$

It is not difficult to see that $\alpha=N / 2$ for some integer $N \leq 2$ (otherwise all $f_{\alpha, j}\left(m_{1}, \ldots, m_{n}\right)$ vanish). For any fixed eigenvalue $\alpha$, we now calculate the explicit expression of the corresponding eigen function $f_{\alpha}\left(m_{1}, \ldots, m_{n}\right)$. There are three cases.

Case 1: $m$ is such that $2 \int_{0}^{1} \gamma^{m / 2+\alpha} d \gamma \neq 1$ and $2 \int_{0}^{1} \gamma^{m / 2+1+\alpha} d \gamma \neq 1$. In this case we get that

$$
f_{\alpha}^{(j)}\left(m_{1}, \ldots, m_{n}\right)=0, \quad \forall m_{1}+\cdots+m_{n}=m, \forall j=1, \ldots, n .
$$

Case 2: $2 \int_{0}^{1} \gamma^{m / 2+\alpha} d \gamma=1$ or equivalently $m=2-2 \alpha$. Then,

$$
f_{\alpha, j}\left(m_{1}, \ldots, m_{n}\right)=C_{\alpha}\left(m_{1}, \ldots, m_{n}\right) x^{(j)}, \quad \forall m_{1}+\cdots+m_{n}=m,
$$

where $C_{\alpha}\left(m_{1}, \ldots, m_{n}\right)$ is an arbitrary constant depending only on $\alpha$ and $\left(m_{1}, \ldots, m_{n}\right)$.

Case 3: $2 \int_{0}^{1} \gamma^{m / 2+\alpha+1} d \gamma=1$ or equivalently $m=-2 \alpha$. In this case $f_{\alpha}^{(j)}\left(m_{1}, \ldots, m_{n}\right)$ must satisfy the constraint:

$$
\sum_{j=1}^{n} f_{\alpha}^{(j)}\left(m_{1}, \ldots, m_{n}\right)=0
$$


Concluding from the above three cases, we now formulate our main theorem about the spectrum of the linearized operator.

TheOREM 3.1. The spectrum of the operator $\mathcal{A}$ consists of the following eigen-values

$$
\operatorname{spec}(\mathcal{A})=\left\{1, \frac{1}{2}, 0,-\frac{m}{2}, m \geq 1\right\} .
$$

The first eigen-values have multiplicities $\nu_{1}=1, \nu_{\frac{1}{2}}=n-1, \nu_{0}=$ $\frac{n^{2}+3 n-2}{2}$.

The system of eigenfunctions is complete in the following sense. Let $\Gamma^{(s)}$ be the stable linear subspace of $\Delta$ generated by all eigenfunctions with $\Re(\lambda)<0, \Gamma^{(u)}$ be the unstable subspace generated by all eigenfunctions with eigenvalues $\lambda>0$, and $\Gamma^{(n)}$ be the neutral subspace generated by all eigenfunctions with eigenvalue $\lambda=0$. Then $\operatorname{dim} \Gamma^{(u)}=n$, $\operatorname{dim} \Gamma^{(n)}=\frac{n^{2}+3 n-2}{2}$ and

$$
\Delta=\Gamma^{(u)}+\Gamma^{(n)}+\Gamma^{(s)} .
$$

Proof. By previous arguments, we discuss the spectrum separately in the following three cases.

1 ${ }^{\circ}$ unstable spectrum: $\quad \alpha=1,1 / 2$.

a) $\alpha=1$. In this case we have $f_{1}^{(j)}\left(m_{1}, \ldots, m_{n}\right)=0$ if $m_{1}+\cdots+$ $m_{n}>0$ and $f^{(j)}(0, \ldots, 0)=C x^{(j)}, j=1, \ldots, n$. Clearly this gives $\nu_{1}=1$.

b) $\alpha=1 / 2$. In this case $f_{1 / 2}^{(j)}\left(m_{1}, \ldots, m_{n}\right)=0$ if $m_{1}+\cdots+m_{n} \neq$ 1. And for $m_{1}+\cdots+m_{n}=1$ we have $f_{1 / 2}^{(j)}\left(m_{1}, \ldots, m_{n}\right)=$ $C_{1 / 2}\left(m_{1}, \ldots, m_{n}\right) x^{(j)}, j=1, \ldots, n$. This gives $\nu_{1 / 2}=n-1$.

This gives $\operatorname{dim} \Gamma^{(u)}=n$.

$2^{\circ}$ neutral spectrum: Here we have $\alpha=0$, and two cases.

a) $m_{1}+\cdots+m_{n}=2$. In this case we get $f_{0}^{(j)}\left(m_{1}, \ldots, m_{n}\right)=$ $C_{0}\left(m_{1}, \ldots, m_{n}\right) x^{(j)}, j=1, \ldots, n$. This gives $\frac{(n+1) n}{2}$ eigenvectors.

b) $m_{1}+\cdots+m_{n}=0$. In this case we get $\sum_{j=1}^{n} f_{0}^{(j)}(0, \ldots, 0)=0$, $j=1, \ldots, n$. This gives $n-1$ eigen-vectors.

Putting all two cases together, we see that $\operatorname{dim} \Gamma^{(n)}=\frac{n^{2}+3 n-2}{2}$.

$3^{\circ}$ stable spectrum: $\quad \alpha<0$.

There are two cases.

Case 1: $m_{1}+\cdots+m_{n}=2-2 \alpha$. In this case We have $f_{\alpha}^{(j)}\left(m_{1}, \ldots, m_{n}\right)=$ $C x^{(j)}, 1 \leq j \leq n$. There are $\left(\begin{array}{c}2-2 \alpha+n+1 \\ 2-2 \alpha\end{array}\right)$ such eigen-vectors. 
Case 2: $m_{1}+\cdots+m_{n}=-2 \alpha$. In this case the constraint is

$$
\sum_{j=1}^{n} f_{\alpha}^{(j)}\left(m_{1}, \ldots, m_{n}\right)=0 \quad 1 \leq j \leq n .
$$

There are $(n-1)\left(\begin{array}{c}-2 \alpha+n-1 \\ -2 \alpha\end{array}\right)$ such eigen-vectors.

We now prove the completeness of the set of our eigen functions. Fix $\left(m_{1}, \ldots, m_{n}\right)$ and consider an arbitrary function $G$ having only the nonzero coefficients $g^{(j)}\left(m_{1}, \ldots, m_{n}\right), 1 \leq j \leq n$. We shall show that $G$ can be expressed by a finite linear combination of our eigen functions. There are two cases.

Case 1: $m_{1}=\cdots=m_{n}=0$. From the discussions above we see that $\alpha=1$ has an eigen vector $f_{\alpha=1}^{(j)}(0, \ldots, 0)=C x_{j}$ which is parallel to the vector $x$, and $\alpha=0$ has eigen vectors satisfying $\sum_{j=1}^{n} f_{\alpha=0}^{(j)}(0, \ldots, 0)$ $=0$ which span the space complement to the vector $x$. It is clear that $g^{(j)}$ is in the linear span of these eigen vectors.

Case 2: $m_{1}+\cdots+m_{n}=m \geq 1$. From previous discussions we see that $\alpha=\frac{2-m}{2}$ has an eigenvector $f_{\alpha=\frac{2-m}{2}}^{(j)}\left(m_{1}, \ldots, m_{n}\right)$ parallel to the vector $x$, and $\alpha=-\frac{m}{2}$ has eigen vectors spanning the space complement to the vector $x$. Again it is clear that $g^{(j)}\left(m_{1}, \ldots, m_{n}\right)$ is in the linear span of these eigen vectors.

Concluding from the above 2 cases, we see that our eigen vectors form a basis for the Gaussian weighted space $L^{2}\left(R^{n}\right)^{n}$. The theorem is proved.

\section{The Choice of Initial Conditions and the Initial Part of the Inductive Procedure}

As in $[\mathbf{L S}]$, the equation (11) for the fixed point which was derived in $\S 2$ is non-typical from the point of view of the renormalization group theory because it contains the integration over $\gamma, 0 \leq \gamma \leq 1$. On the other hand, since we consider the Cauchy problem for (1) we are given only the initial condition $v(k, 0)$ which produces through the recurrent relations $(4),(5),(6)$ the whole set of functions $g_{r}(k, s)$. For large $p$ and $r \leq p$ they can be considered as depending on $\gamma=\frac{r}{p}$ and our procedure is organized in such a way that for $\gamma$ which are away from zero $\tilde{g}_{r}$ are close to their limits.

We take $k^{(0)}$ which will be assumed to be sufficiently large, introduce the neighborhood

$$
A_{1}=\left\{k:\left|k-\kappa^{(0)}\right| \leq D_{1} \sqrt{k^{(0)} \ln k^{(0)}}\right\}
$$


where $\kappa^{(0)}=k^{(0)}(1, \ldots, 1)$ and $D_{1}$ is also sufficiently large. Our initial conditions will be zero outside $A_{1}$. Inside $A_{1}$ they have the form

$$
\begin{array}{r}
v(k, 0)=\frac{1}{(2 \pi)^{n / 2}} \exp \left\{-\frac{|Y|^{2}}{2}\right\}\left(H^{(0)}(Y)+\sum_{j=1}^{l^{(u)}} b_{j}^{(u)} \Phi_{j}^{(u)}(Y)+\right. \\
\left.\sum_{j^{\prime}=1}^{l^{(n)}} b_{j^{\prime}}^{(n)} \Phi_{j^{\prime}}^{(n)}(Y)+\Phi\left(Y ; b^{(u)}, b^{(n)}\right)\right)
\end{array}
$$

In this expression $k=\kappa^{(0)}+\sqrt{k^{(0)}} Y, H^{(0)}(Y)$ is the fixed point of our renormalization group with $\sigma^{(1)}=\cdots=\sigma^{(n)}=1$ and $x^{(1)}=\cdots=$ $x^{(n)}=2 / n($ see $\S 2), l^{(u)}=n$ and $l^{(n)}=\frac{n^{2}+3 n-2}{2}($ see $\S 3)$. Also $\Phi_{j}^{(u)}$, $\Phi_{j^{\prime}}^{(n)}$ are unstable and neutral eigen-functions of the linearized group corresponding to $H^{(0)}$ (see $\left.\S 3\right), b_{j}^{(u)}$ and $b_{j^{\prime}}^{(n)}$ are our main parameters. We assume that their values satisfy the inequalities:

$$
-\rho_{1} \leq b_{j}^{(u)}, b_{j^{\prime}}^{(n)} \leq \rho_{1},
$$

where $\rho_{1}$ is a positive constant. Our numerical studies show that it is enough to take $\rho_{1}=3 / 4$. Each function $\Phi\left(Y ; b^{(u)}, b^{(n)}\right), b^{(u)}=\left\{b_{j}^{(u)}\right\}$, $b^{(n)}=\left\{b_{j^{\prime}}^{(n)}\right\}$ is small in the sense that it satisfies the inequalities

$$
\sup _{Y, b}\left|\Phi\left(Y ; b^{(u)}, b^{(n)}\right)\right| \leq D_{2},
$$

$\sup \left\|\Phi\left(Y ; \bar{b}^{(u)}, \bar{b}^{(n)}\right)-\Phi\left(Y ; \overline{\bar{b}}^{(u)}, \overline{\bar{b}}^{(n)}\right)\right\| \leq D_{2}\left(\left|\bar{b}^{(u)}-\overline{\bar{b}}^{(u)}\right|+\left|\bar{b}^{(n)}-\overline{\bar{b}}^{(n)}\right|\right)$.

Due to the presence of $b^{(u)}, b^{(n)}$, we have $l=l^{(u)}+l^{(n)}=\frac{n^{2}+5 n-2}{2}$ parameter families of initial conditions, due to the presence of $\Phi$ we have an open set in the space of such families.

Let

$$
A_{r}=\left\{k:\left|k-r \kappa^{(0)}\right| \leq D_{1} \sqrt{r k^{(0)} \ln \left(r k^{(0)}\right)}\right\},
$$

and the variable $Y$ be such that $k=r \kappa^{(0)}+\sqrt{r k^{(0)}} Y$. Assume that for some $p$ and all $r<p,|Y| \leq D_{1} \sqrt{\ln \left(r k^{(0)}\right)}$

$$
g_{r}\left(r \kappa^{(0)}+\sqrt{r k^{(0)}} Y, s\right)=Z \cdot \Lambda_{r}(s) \cdot r^{\frac{4-n}{2}} \cdot h_{r}(Y, s)
$$

and

$$
h_{r}(Y, s)=\frac{1}{(2 \pi)^{n / 2}} \exp \left\{-\frac{|Y|^{2}}{2}\right\}\left(H^{(0)}(Y)+\delta^{(r)}(Y, s)\right),
$$

where $Z$ is a constant to be specified later and $\Lambda_{r}(s)$ is a function of $s$ for each $r$. 
Denote $p_{0}=N$ where $N$ is an integer. Actually we will take $N=50$. The initial part of our procedure goes for $p \leq p_{0}$. It is discussed in this section. The part corresponding to $p>p_{0}$ is discussed in $\S 5$.

Returning back to (6) take the term with some $p_{1}, p_{2}, p_{1}+p_{2}=p$ and introduce new variable of integration $Y^{\prime}$ where $k^{\prime}=p_{2} \kappa^{(0)}+\sqrt{p k^{(0)}} Y^{\prime}$. Introduce also the variables $\theta_{1}, \theta_{2}, 0 \leq \theta_{1} \leq\left(p_{1} k^{(0)}\right)^{2}, 0 \leq \theta_{2} \leq\left(p_{2} k^{(0)}\right)^{2}$ where $s_{1}=s\left(1-\frac{\theta_{1}}{\left(p_{1} k^{(0)}\right)^{2}}\right), s_{2}=s\left(1-\frac{\theta_{2}}{\left(p_{2} k^{(0)}\right)^{2}}\right)$.

Then from (6)

$$
\begin{aligned}
\frac{1}{Z} & \cdot \Lambda_{p} \cdot p^{\frac{4-n}{2}} h_{p}(Y, s) \\
= & \left(p k^{(0)}\right)^{\frac{n+2}{2}} \sum_{p_{1}+p_{2}=p}\left(p_{1} p_{2}\right)^{\frac{4-n}{2}} \int_{0}^{p_{1}^{2}} d \theta_{1} \cdot \\
& \cdot \int_{0}^{p_{2}^{2}} d \theta_{2} \frac{1}{p_{1}^{2} p_{2}^{2}} \Lambda_{p_{1}}\left(\left(1-\frac{\theta_{1}}{p_{1}^{2}}\right) s\right) \Lambda_{p_{2}}\left(\left(1-\frac{\theta_{2}}{p_{2}^{2}}\right) s\right) \cdot \\
& \cdot \int_{R^{n}}\left\langle h_{p_{1}}\left(\frac{Y-Y^{\prime}}{\sqrt{\gamma}},\left(1-\frac{\theta_{1}}{p_{1}^{2}}\right) s\right), \frac{p_{2}}{p} \kappa^{(0,0)}+\frac{Y^{\prime}}{\sqrt{p k^{(0)}}}\right\rangle . \\
& \cdot h_{p_{2}}\left(\frac{Y^{\prime}}{\sqrt{1-\gamma}},\left(1-\frac{\theta_{1}}{p_{1}^{2}}\right) s\right) \cdot \\
& \cdot e^{-\theta_{1}\left|\kappa^{(0)}+\sqrt{k^{(0)}} \frac{Y-Y^{\prime}}{\sqrt{p} \gamma}\right|^{2}-\theta_{2}\left|\kappa^{(0)}+\sqrt{k^{(0)}} \frac{Y^{\prime}}{\sqrt{p}(1-\gamma)}\right|^{2} d^{n} Y^{\prime},}
\end{aligned}
$$

or in a slightly simpler form,

$$
\begin{aligned}
& \frac{\left(k^{(0)}\right)^{-\frac{n+2}{2}}}{Z} \cdot \Lambda_{p} \cdot h_{p}(Y, s) \\
= & \frac{1}{p} \sum_{p_{1}+p_{2}=p}(\gamma(1-\gamma))^{-n / 2} \int_{0}^{p_{1}^{2}} d \theta_{1} . \\
& \cdot \int_{0}^{p_{2}^{2}} d \theta_{2} \Lambda_{p_{1}}\left(\left(1-\frac{\theta_{1}}{p_{1}^{2}}\right) s\right) \Lambda_{p_{2}}\left(\left(1-\frac{\theta_{2}}{p_{2}^{2}}\right) s\right) \cdot \\
& \cdot \int_{R^{n}}\left\langle h_{p_{1}}\left(\frac{Y-Y^{\prime}}{\sqrt{\gamma}},\left(1-\frac{\theta_{1}}{p_{1}^{2}}\right) s\right), \frac{p_{2}}{p} \kappa^{(0,0)}+\frac{Y^{\prime}}{\sqrt{p k^{(0)}}}\right\rangle . \\
& \cdot h_{p_{2}}\left(\frac{Y^{\prime}}{\sqrt{1-\gamma}},\left(1-\frac{\theta_{1}}{p_{1}^{2}}\right) s\right) \cdot \\
& \cdot e^{-\theta_{1}\left|\kappa^{(0)}+\sqrt{k^{(0)}} \frac{Y-Y^{\prime}}{\sqrt{p} \gamma}\right|^{2}-\theta_{2}\left|\kappa^{(0)}+\sqrt{k^{(0)}} \frac{Y^{\prime}}{\sqrt{p}(1-\gamma)}\right|^{2} d^{n} Y^{\prime} .}
\end{aligned}
$$

Here $\gamma=\frac{p_{1}}{p}$. Now we shall modify (22) similar to what we did in $\S 2$. As in $[\mathbf{L S}]$, the modification consists of two steps. 
Step 1. All terms $s\left(1-\frac{\theta_{1}}{\left(p_{1} k^{(0)}\right)^{2}}\right), s\left(1-\frac{\theta_{2}}{\left(p_{2} k^{(0)}\right)^{2}}\right)$ are replaced by $s$.

Step 2. In the main order of magnitude,

$$
\left\langle h_{p_{1}}\left(\frac{Y-Y^{\prime}}{\sqrt{\gamma}}, s\right), \frac{p_{2}}{p} \kappa^{(0,0)}+\frac{Y^{\prime}}{\sqrt{p k^{(0)}}}\right\rangle=\frac{p_{2}}{p} \sum_{j=1}^{n} h_{p_{1}}^{(j)}\left(\frac{Y-Y^{\prime}}{\sqrt{\gamma}}, s\right) .
$$

We take $Z=\left(k^{(0)}\right)^{\frac{6-n}{2}}$ and choose the initial interval on the time axis in the form $S^{(1)}=\left[\frac{1}{4\left(k^{(0)}\right)^{2}}, \frac{3}{8\left(k^{(0)}\right)^{2}}\right]$. Due to our choice of $S^{(1)}$ the product $s\left(k^{(0)}\right)^{2}=O(1)$. During the first part of our procedure $S^{(p)}=S^{(1)}, p \leq p_{0}$. Now we derive the recurrent formulas for $\Lambda_{p}$. For our fixed point $H^{(0)}$, the integral in (22) containing $H^{(0)}$ gives us the product of $H^{(0)}$, the Gaussian term and a polynomial in $\gamma$. The function $H^{(0)}$ and the Gaussian term can be taken out of the summation in $\gamma$. After symmetrizing the summation in $\gamma$, we get the following recurrent system for $\Lambda_{p}(s)$ :

$$
\Lambda_{p}(s)=\sum_{p_{1}+p_{2}=p} \frac{1}{p} \cdot \Lambda_{p_{1}}(s) \Lambda_{p_{2}}(s) \cdot\left(1-e^{-s\left(p_{1} k^{(0)}\right)^{2}}\right) \cdot\left(1-e^{-s\left(p_{2} k^{(0)}\right)^{2}}\right) .
$$

In $[\mathbf{L S}]$, we have a similar (but more complicated) recurrent system for $\Lambda_{p}(s)$. It follows from $[\mathbf{L i}]$, [Si $\left.\mathbf{3}\right]$ that the asymptotics of $\Lambda_{p}(s)$ is given by,

$$
\Lambda_{p}(s)=\Lambda(s)^{p} \cdot\left(1+O\left(\frac{1}{p^{3 / 2}}\right)\right)
$$

where $\Lambda(s)>0$ is a limiting constant independent of $p$. This result will be used in the proof of the main theorem.

Now we shall discuss the behavior of all remainders $\delta^{(r)}, r<p$. We make the following inductive assumption:

$$
\begin{aligned}
\delta^{(r)}(Y, s)= & \sum_{j=1}^{l^{(u)}}\left(b_{j, r}^{(u)}+\beta_{j, r}^{(u)}\right) \gamma_{j}^{\alpha_{j}^{(u)}} \Phi_{j}^{(u)}(Y) \\
& +\sum_{j^{\prime}=1}^{l^{(n)}}\left(b_{j^{\prime}, r}^{(n)}+\beta_{j^{\prime}, r}^{(n)}\right) \Phi_{j^{\prime}}^{(n)}(Y)+\Phi_{r}^{(s t)}(Y, s), \quad \gamma=\frac{r}{p-1} .
\end{aligned}
$$

Here $b_{j, r}^{(u)}=(p-1)^{\alpha_{j}} b_{j}^{(u)} \gamma^{\alpha_{j}^{(u)}}, b_{j^{\prime}, r}^{(n)}=b_{j^{\prime}}^{(n)}$, and the corrections $\beta_{j, r}^{(u)}$, $\beta_{j^{\prime}, r}^{(n)}$ are small compared to $b_{j, r}^{(u)}, b_{j^{\prime}, r}^{(n)}$ respectively. Also $\left\{\Phi_{r}^{(s t)}(Y, s)\right\}$, $1 \leq r<p$ belongs to the stable subspace of our fixed point. 
As we go from $p-1$ to $p$, the variable $\gamma=\frac{r}{p-1}$ changes to $\gamma^{\prime}=\frac{r}{p}=$ $\gamma \cdot \frac{p-1}{p}$. Therefore

$$
\begin{aligned}
& \left(b_{j, r}^{(u)}+\beta_{j, r}^{(u)}\right) \gamma^{\alpha_{j}^{(u)}} \Phi_{j}^{(u)} \\
& =\left((p-1)^{\alpha_{j}^{(u)}} b_{j}^{(u)}+\beta_{j, r}^{(u)}\right) \cdot\left(\frac{p}{p-1}\right)^{\alpha_{j}^{(u)}} \cdot\left(\gamma^{\prime}\right)^{\alpha_{j}^{(u)}} \Phi_{j}^{(u)} \\
& =\left(p^{\alpha_{j}^{(u)}} b_{j}^{(u)}+\left(\frac{p}{p-1}\right)^{\alpha_{j}^{(u)}} \cdot \beta_{j, r}^{(u)}\right) \cdot\left(\gamma^{\prime}\right)^{\alpha_{j}^{(u)}} \Phi_{j}^{(u)} \cdot
\end{aligned}
$$

The formulas for the part involving the neutral eigen-functions are similar and even simpler because $\alpha_{j^{\prime}}^{(n)}=0$. Thus the main terms in the expressions containing unstable and neutral eigen-values preserve their form. The norm of $\left\{\Phi_{r}^{s t}\right\}$ decreases.

In the initial part of our procedure with $p \leq p_{0}$ we use the discrete recurrent formulas and get small corrections $\beta_{j, r}^{(u)}, \beta_{j^{\prime}, r}^{(n)}$ and $\Phi^{(s t)}$. We consider four types of corrections.

$\left.a_{1}\right)$ In the expression for $\delta^{(p)}(Y, s)$ there are terms which depend linearly on all $\delta^{(r)}(Y, s)$. Especially important are the terms $p^{\alpha_{j}^{(u)}} b_{j}^{(u)}(\gamma)^{\alpha_{j}^{(u)}}, \Phi_{j}^{(u)}$ and $b_{j^{\prime}}^{(n)} \Phi_{j^{\prime}}^{(n)}$. In the limiting regime $p \rightarrow$ $\infty$ they produce the integral over $\gamma$ which gives

$$
p^{\alpha_{j}^{(u)}} b_{j}^{(u)} \cdot\left(1+\frac{1}{p}\right)^{\alpha_{j}^{(u)}} \Phi_{j}^{(u)}=(p+1)^{\alpha_{j}^{(u)}} b_{j}^{(u)} \Phi_{j}^{(u)},
$$

in view of the definition of the eigenfunctions (see $\S 3$ ) and the condition $\gamma^{\prime}=1$. The same statement holds true for the neutral eigen-functions.

However for finite $p$, the sums over $\gamma$ differ from the corresponding integrals. The difference produces some corrections which we expand according to our decomposition of the whole space onto unstable, neutral subspaces and the stable subspace. Corresponding terms are denoted as $\beta_{j, p, 1}^{(u)}, \beta_{j^{\prime}, p, 1}^{(n)}$ and $\Phi_{p, 1}^{(s t)}$. These corrections decay as $O\left(\frac{1}{p}\right)$.

$a_{2}$ ) The term which contains all corrections arising during the two steps of our procedure (see above). All these corrections depend on $k^{(0)}$ and are smaller than $\frac{1}{\left(k^{(0)}\right)^{\mu_{1}}}$ for some positive constant $\mu_{1}$.

$\left.a_{3}\right)$ The term $\tilde{\beta}_{p}$ which is a linear function of all $\beta_{j, r}^{(u)}, \beta_{j, r}^{(n)}, 1 \leq r \leq$ $p-1$. We use the Hilbert space $X^{(p)}$ consisting of functions 
$f=\left\{f_{r}(Y), 1 \leq r<p\right\}$ equiped with the norm

$$
\|f\|^{2}=\frac{1}{p-1} \sum_{r=1}^{p-1}\left\|f_{r}(Y)\right\|_{L^{2}}^{2}
$$

where $\|\cdot\|_{L^{2}}$ is the norm in the space of square-integrable functions of $Y$. It follows easily from $\S 3$ that for some constant $C_{1}$

$$
\left\|\tilde{\beta}_{p}\right\|_{L^{2}} \leq C_{1}\left(\sum_{j=1}^{l^{(u)}}\left\|\beta_{j}^{(u)}\right\|+\sum_{j^{\prime}=1}^{l^{(n)}}\left\|\beta_{j^{\prime}}^{(n)}\right\|\right) .
$$

Therefore

$$
\begin{aligned}
& \left\|\left\{\beta_{j, r}^{(u)}, \beta_{j^{\prime}, r}^{(n)}\right\}, 1 \leq r \leq p-1, \tilde{\beta}_{p}\right\|_{X^{(p+1)}}^{2} \\
& =\frac{1}{p} \sum_{r=1}^{p}\left(\left\|\beta_{j, r}^{(u)}\right\|^{2}+\left\|\beta_{j^{\prime}, r}^{(n)}\right\|^{2}\right) \\
& =\frac{p-1}{p}\left\|\left\{\beta_{j, r}^{(u)}, \beta_{j^{\prime}, r}^{(n)}, 1 \leq r \leq p-1\right\}\right\|_{X^{(p-1}}^{2}+\frac{1}{p}\left\|\tilde{\beta}_{p}\right\|^{2} \\
& \leq\left\|\left\{\beta_{j, r}^{(u)}, \beta_{j^{\prime}, r}^{(n)}, 1 \leq r \leq p-1\right\}\right\|^{2} \cdot\left(\frac{p-1}{p}+\frac{C_{1}}{p}\right) \\
& \leq\left\|\left\{\beta_{j, r}^{(u)}, \beta_{j^{\prime}, r}^{(n)}, 1 \leq r \leq p-1\right\}\right\|^{2} \cdot\left(1+\frac{C_{2}}{p}\right)
\end{aligned}
$$

for another constant $C_{2}$. Iterating this estimate we get

$$
\left\|\left\{\beta_{j, r}^{(u)}, \beta_{j^{\prime}, r}^{(n)}, 1 \leq r \leq p\right\}\right\| \leq\left\|\beta_{j, 1}^{(u)}, \beta_{j^{\prime}, 1}^{(n)}\right\| \cdot \prod_{q=1}^{p}\left(1+\frac{C_{2}}{q}\right) .
$$

The first term $\left\|\beta_{j, 1}^{(u)}, \beta_{j^{\prime}, 1}^{(n)}\right\| \leq \frac{1}{\left(k^{(0)}\right)^{\mu_{2}}}$ for another constant $\mu_{2}$ and $\prod_{q=1}^{p}\left(1+\frac{C_{2}}{q}\right) \leq p_{0}^{C_{3}}$ for another constant $C_{3}$. This gives the estimate of the linear part.

$a_{4}$ ) All terms which are quadratic functions of all remainders. Since all previous terms were already estimated the quadratic terms are much smaller than the previous ones.

The sum of all these terms gives $\beta_{j, p}^{(u)}, \beta_{j^{\prime}, p}^{(n)}, \Phi_{p}^{s t}$.

We take $N=50$. For all $p \leq 50$ all remainders were found numerically with the help of computer using exact recurrent relations (7). At $N=50$ we make the first rescaling. Put $b_{j}^{(u, 1)}=p^{\alpha_{j}} b_{j}^{(u)}+\beta_{j, p}^{(u)}$, for $1 \leq j \leq l^{(u)}$ and $b_{j^{\prime}}^{(n, 1)}=b_{j^{\prime}}^{(n)}+\beta_{j^{\prime}, p}^{(n)}$ for $1 \leq j^{\prime} \leq l^{(n)}$ and $p=50$. These are our new rescaled variables. All previous expressions for $\delta^{(r)}(Y, s)$, $r<N$ can be written as functions of these new variables:

$$
b_{j}^{(u)} p^{\alpha_{j}}+\beta_{j, r}^{(u)}=b_{j}^{(u, 1)}+\beta_{j, r}^{(u, 1)}, \quad 1 \leq j \leq l^{(u)},
$$


where $\beta_{j, r}^{(u, 1)}=\beta_{j, r}^{(u)}-\beta_{j, p}^{(u)}$ and

$$
b_{j^{\prime}}^{n)}+\beta_{j^{\prime}, r}^{(n)}=\beta_{j^{\prime}, 1}^{(n, 1)}+\beta_{j^{\prime}, r}^{(n, 1)}, \quad 1 \leq j^{\prime} \leq l^{(n)},
$$

where $\beta_{j^{\prime}, r}^{(n, 1)}=\beta_{j^{\prime}, r}^{(u)}-\beta_{j^{\prime}, p}^{(n)}$. The change in the expression for $\Phi_{r}^{(s t)}$ is just the change of variables $b_{j}^{(u)}, b_{j^{\prime}}^{(n)}$. Numerically it was shown that $\rho_{1}$ can be chosen in such a way that the set $b_{j}^{(u)}, b_{j^{\prime}}^{(n)}$ for which $-\frac{\rho_{1}}{2} \leq$ $b_{j}^{(u, 1)}, b_{j^{\prime}}^{(n, 1)} \leq \frac{\rho_{1}}{2}$ is contained in the original set $-\rho_{1} \leq b_{j}^{(u)}, b_{j^{\prime}}^{(n)} \leq \rho_{1}$. We use this procedure till $p=p_{0}=N$. The procedure for $p>p_{0}$ will be discussed in $\S 6$.

\section{The List of Remainders and Their Estimates}

In the beginning of $\S 4$ we described $\left(n^{2}+5 n-2\right) / 2$-parameter families of initial conditions which we consider in this paper. We mentioned above that for each $\mathrm{p}$ we have an interval $S^{(p)}=\left[S_{-}^{(p)}, S_{+}^{(p)}\right]$ on the time axis. Actually these intervals will be changed only when $p=$ $p_{n}=(1+\epsilon)^{n}$ where $\epsilon>0$ is a constant. Therefore we shall write $S^{(n)}=\left[S_{-}^{\left(p_{n}\right)}, S_{+}^{\left(p_{n}\right)}\right]$ and hope that there will be no confusion.

In this and the next section we consider $p>p_{0}$. Each function $\tilde{g}_{r}(Y, s), r<p$, has the following representation:

in the domain $|Y| \leq D_{1} \sqrt{\ln r k^{(0)}}$,

$$
\tilde{g}_{r}(Y, s)=Z \cdot \Lambda_{r}(s) \cdot r^{\frac{4-n}{2}} \cdot \frac{1}{(2 \pi)^{n / 2}} \cdot e^{-\frac{|Y|^{2}}{2}}\left(H^{(0)}(Y)+\delta^{(r)}(Y, s)\right) .
$$

in the domain $|Y|>D_{1} \sqrt{\ln \left(r k^{(0)}\right)}$ :

$$
\left|\tilde{g}_{r}(Y, s)\right| \leq \frac{B_{1} \Lambda_{r}(s)}{r^{\lambda_{1}}}
$$

for some constants $\lambda_{1}>0, B_{1}>0$. We use the formula (7) to get $\tilde{g}^{(p)}(Y, s)$. New remainders appear in one of the following ways.

Type I. The recurrent relation (7) does not coincide with the equation for the fixed point and actually is some perturbation of this equation. The difference produces some remainders which tend to zero as $p \rightarrow \infty$.

Type II. For the limiting equation all eigen-vectors in the linear approximation are multiplied by some constant. In the equation (7) it is no longer true and the difference generate some remainders. (see also $\S 6$ ).

Type III. The remainders which are quadratic functions of all previous remainders. 
5.1. The Remainders of Type I. We call the domain $A$ the set $\left\{|Y| \leq D_{1} \sqrt{\ln \left(r k^{(0)}\right)}\right\}$ and the domain $B$ the set $\left\{|Y|>D_{1} \sqrt{\ln \left(r k^{(0)}\right)}\right\}$. The estimates will be done separately in each domain. We include the first, the second and the last two terms in (7) in the remainders. We shall estimate only the first one, the others are estimated in the same way.

Domain A: We have

$$
\begin{aligned}
\beta_{p}^{(1)}(Y, s) \\
=\left((p+1) k^{(0)}\right)^{\frac{n+2}{2}} \cdot \frac{1}{p^{2}} \cdot \int_{0}^{p^{2}} d \theta_{2} \cdot \\
\cdot \int_{R^{n}}\left\langle v\left(k^{(0)}+\sqrt{(p+1) k^{(0)}}\left(Y-Y^{\prime}\right) ; b\right) \kappa^{(0,0)}+\frac{Y}{\sqrt{p+1}}\right\rangle \cdot \\
\cdot \tilde{g}_{p}\left(Y^{\prime},\left(1-\frac{\theta_{2}}{p^{2}}\right) s\right) \cdot \\
\quad \cdot \exp \left\{-\left|k^{(0)}+\left(Y-Y^{\prime}\right) \sqrt{p+1}\right|^{2}-\frac{\theta_{2}}{p^{2}}\left|k^{(0)} p+Y^{\prime} \sqrt{p+1}\right|^{2}\right\} d^{n} Y^{\prime} .
\end{aligned}
$$

Here $b$ means the collection of all parameters in the definition of $v(k ; 0)$. The main contribution to the integral comes from $Y-Y^{\prime}=O\left(\frac{1}{\sqrt{p+1}}\right)$. In this domain in the main order of magnitude

$$
\left\langle v\left(k^{(0)}+\left(Y-Y^{\prime}\right) \sqrt{p+1}, 0 ; b\right), \kappa^{(0,0)}\right\rangle=O(1)
$$

Assuming that $v\left(k^{(0)}+\left(Y-Y^{\prime}\right) \sqrt{p+1}, 0 ; b\right)$ is differentiable w.r.t the first three variables we see that the inner product

$$
\left\langle v\left(k^{(0)}+\left(Y-Y^{\prime}\right) \sqrt{p+1}, 0 ; b\right), \kappa^{(0,0)}+\frac{Y}{\sqrt{p+1}}\right\rangle
$$

is of order $O(1)$. For $\tilde{g}_{p}$ we can write using our inductive assumptions

$$
\begin{aligned}
& \tilde{g}_{p}\left(Y^{\prime},\left(1-\frac{\theta_{2}}{p^{2}}\right) s\right) \\
& =Z \cdot \Lambda_{p}(s) \cdot p^{\frac{4-n}{2}} \cdot \frac{1}{(2 \pi)^{n / 2}} \cdot e^{-\frac{|Y|^{2}}{2}} \cdot \mathcal{H}^{(p)}\left(Y^{\prime},\left(1-\frac{\theta_{2}}{p^{2}}\right) s\right) .
\end{aligned}
$$

where $\left.\mathcal{H}^{(p)}(Y, s)=H^{(0)}(Y, s)+\delta^{(p)}(Y, s)\right)$. Also

$$
\exp \left\{-\frac{\theta_{2}}{p^{2}}\left|k^{(0)} p+Y^{\prime} \sqrt{p+1}\right|^{2}\right\}=\exp \left\{-\theta_{2}\left|k^{(0)}+\frac{Y^{\prime} \sqrt{p+1}}{p}\right|^{2}\right\}
$$

and in the main order of magnitude the integration over $\theta_{2}$ does not depend on $Y^{\prime}$. Thus we can write

$$
\left|\beta_{p}^{(1)}(Y, s)\right| \leq D_{4} \cdot \frac{1}{p} \cdot p^{\frac{4-n}{2}} \cdot \Lambda_{p}(s) \cdot e^{-\frac{|Y|^{2}}{2}} .
$$


Here and later various constants whose exact values play no role in the arguments will be denoted by the letter $D$ with indices. Since in the expression for $\tilde{g}_{p+1}$ we have the factors $\Lambda_{p+1} \cdot(p+1)^{\frac{4-n}{2}} \cdot e^{-\frac{|Y|^{2}}{2}}$, the estimate $(25)$ shows that $\left|\beta_{p}^{(1)}(Y, s)\right|$ is much smaller than $\tilde{g}_{p+1}$ by a factor of $O\left(\frac{1}{p}\right)$. This is good enough for our purposes. We do not discuss the errors which follow from the fact that the expressions in the previous formulas depend on $\theta_{2}$.

Domain B: The smallness of $\beta_{p}^{(1)}(Y, s)$ in this case follows easily from several inequalities and arguments.

$1^{\circ}: \quad|Y| \leq D_{4} \sqrt{p k^{(0)}}$ because $|k| \leq D_{5} p k^{(0)}$.

$2^{\circ}: \quad\left|Y-Y^{\prime}\right| \leq D_{6} \sqrt{k^{(0)}}$ because $v(k, 0 ; b)$ has a compact support.

$3^{\circ}: \quad$ If $\left|Y-Y^{\prime}\right| \leq \frac{2 s_{+}}{\sqrt{p}} \quad$ (recall that $S=\left[S_{-}, S_{+}\right]$, we denote here $\left.s_{+}=S_{+}\right)$, then

$$
\exp \left\{-\left|k^{(0)}+\left(Y-Y^{\prime}\right) \sqrt{p+1}\right|^{2}\right\} \leq 1
$$

If $\left|Y-Y^{\prime}\right| \geq \frac{2 s_{+}}{\sqrt{p}}$ then

$$
\exp \left\{-\left|k^{(0)}+\left(Y-Y^{\prime}\right) \sqrt{p+1}\right|^{2}\right\} \leq \exp \left\{-\frac{s_{+}}{4}\left|Y-Y^{\prime}\right|^{2}\right\} .
$$

$4^{\circ}: \quad$ If $\left|Y^{\prime}\right| \geq D_{7} \sqrt{p}$ then

$$
\exp \left\{-\frac{\theta_{2}}{p^{2}}\left|k^{(0)} p+Y^{\prime} \sqrt{p+1}\right|^{2}\right\} \leq \exp \left\{-D_{8} \theta_{2}\right\}
$$

$5^{\circ}: \quad$ If $\left|Y^{\prime}\right| \leq D_{7} \sqrt{p}$ then

$$
\exp \left\{-\frac{\theta_{2}}{p^{2}}\left|k^{(0)} p+Y^{\prime} \sqrt{p+1}\right|^{2}\right\} \leq 1 .
$$

$6^{\circ}$ : We have

$$
\begin{aligned}
& \exp \left\{-\frac{1}{2}|Y|^{2}\right\}=\exp \left\{Y \cdot\left(Y-Y^{\prime}\right)-\frac{1}{2}\left|Y-Y^{\prime}\right|^{2}\right\} . \\
& \text { If }\left|Y-Y^{\prime}\right| \leq \frac{2 s_{+}}{\sqrt{p}} \text { then } \\
& \quad \exp \left\{Y \cdot\left(Y-Y^{\prime}\right)-\frac{1}{2}\left|Y-Y^{\prime}\right|^{2}\right\} \leq D_{8} .
\end{aligned}
$$

If $\left|Y-Y^{\prime}\right|>\frac{2 s_{+}}{\sqrt{p}}$ then we have an integral of the function which is the product of some Gaussian factor and $\left|\mathcal{H}^{(p)}(Y)\right|$. Direct estimate shows as before that in this case

$$
\left|\beta_{p}^{(1)}(Y, s)\right| \leq \Lambda_{p}(s) \cdot p^{\frac{4-n}{2}} \cdot e^{-\frac{|Y|^{2}}{2}} \cdot \frac{D_{8}}{p^{3 / 2}}
$$


which is also good for us.

In the same way one can estimate terms with relatively small $p_{1}$ and $p-p_{1}$ (i.e., $p_{1} \leq \sqrt{p}$ or $p_{1} \geq p-\sqrt{p}$ ). The remainders will be of order $\frac{1}{\sqrt{p_{1}}} \cdot \frac{1}{p}$. The next set of remainders comes from splitting the integration over $\theta$ and $Y^{\prime}$ (see (7) and beginning of $\S 2$ ). We may assume that $p_{1}>\sqrt{p}$ or $p_{1}<p-\sqrt{p}$ because other terms were estimated before.

Put

$$
\begin{aligned}
& \tilde{\tilde{g}}_{p+1}(Y, s) \\
& =\left((p+1) k^{(0)}\right)^{\frac{n+2}{2}} \sum_{\substack{p_{1}+p_{2}=p+1 \\
p_{1}, p_{2}>\sqrt{p}}} \int_{0}^{p_{1}^{2}} d \theta_{1} \int_{0}^{p_{2}^{2}} d \theta_{2} \cdot \frac{1}{p_{1}^{2} p_{2}^{2}} \cdot \\
& \quad \cdot \int_{\mathbb{R}^{3}}\left\langle\tilde{g}_{p_{1}}\left(\frac{Y-Y^{\prime}}{\sqrt{\gamma}},\left(1-\frac{\theta_{1}}{p_{1}^{2}}\right) s\right), k^{(0)}+\frac{Y}{\sqrt{p+1}}\right\rangle \cdot \\
& \cdot \tilde{g}_{p_{2}}\left(\frac{Y^{\prime}}{\sqrt{1-\gamma}},\left(1-\frac{\theta_{2}}{p_{2}^{2}}\right) s\right) \cdot \\
& \exp \left\{-\theta_{1}\left|\kappa^{(0)}+\sqrt{k^{(0)}} \frac{Y-Y^{\prime}}{\sqrt{p+1} \cdot \gamma}\right|^{2}-\theta_{2}\left|\kappa^{(0)}+\frac{\sqrt{k^{(0)}} Y^{\prime}}{\sqrt{p+1}(1-\gamma)}\right|^{2}\right\} d^{n} Y^{\prime} .
\end{aligned}
$$

Using the inductive assumption we can rewrite the last expression as follows:

$$
\begin{aligned}
& \tilde{\tilde{g}}_{p+1}(Y, s) \\
& =\left(k^{(0)}\right)^{\frac{n-6}{2}} Z(s)^{2} \cdot(p+1)^{\frac{4-n}{2}} \sum_{\substack{p_{1}+p_{2}=p+1 \\
p_{1}, p_{2}>\sqrt{p}}} \int_{0}^{p_{1}^{2}} d \theta_{1} \int_{0}^{p_{2}^{2}} d \theta_{2} \\
& \Lambda_{p_{1}} \cdot \Lambda_{p_{2}} \cdot \gamma^{-n / 2}(1-\gamma)^{-n / 2} \cdot \frac{1}{p+1} \cdot \\
& \exp \left\{-\theta_{1}\left|\kappa^{(0)}+\sqrt{k^{(0)}} \frac{Y-Y^{\prime}}{\sqrt{p+1} \cdot \gamma}\right|^{2}-\theta_{2}\left|\kappa^{(0)}+\frac{\sqrt{k^{(0)}} Y^{\prime}}{\sqrt{p+1}(1-\gamma)}\right|^{2}\right\} . \\
& \left\langle\mathcal{H}^{\left(p_{1}\right)}\left(\frac{Y-Y^{\prime}}{\sqrt{\gamma}}, s\left(1-\frac{\theta_{1}}{p_{1}^{2}}\right)\right), \kappa^{(0,0)}+\frac{Y}{\sqrt{p}}\right\rangle \mathcal{H}^{\left(p_{2}\right)}\left(\frac{Y^{\prime}}{\sqrt{1-\gamma}}, s\left(1-\frac{\theta_{2}}{p_{2}^{2}}\right)\right) d^{n} Y^{\prime} .
\end{aligned}
$$

As was explained before, in the Domain A the inner product

$$
\left\langle\mathcal{H}^{\left(p_{1}\right)}\left(\frac{Y-Y^{\prime}}{\sqrt{\gamma}}, s\left(1-\frac{\theta_{1}}{p_{1}^{2}}\right)\right), \kappa^{(0,0)}+\frac{Y}{\sqrt{p}}\right\rangle
$$


takes values $O(1)$ and the remainder can be written in the following form:

$$
\begin{aligned}
& \beta_{p}^{(2)}(Y, s) \\
& =\sum_{\substack{p_{1}+p_{2}=p+1 \\
p_{1}, p_{2}>\sqrt{p}}} \int_{0}^{p_{1}^{2}} d \theta_{1} \int_{0}^{p_{2}^{2}} d \theta_{2} \cdot \gamma^{-n / 2}(1-\gamma)^{-n / 2} \cdot \frac{1}{p+1} \cdot \\
& \exp \left\{-\theta_{1}\left|\kappa^{(0)}+\sqrt{k^{(0)}} \frac{Y-Y^{\prime}}{\sqrt{p+1} \cdot \gamma}\right|^{2}-\theta_{2}\left|\kappa^{(0)}+\frac{\sqrt{k^{(0)}} Y^{\prime}}{\sqrt{p+1}(1-\gamma)}\right|^{2}\right\} . \\
& \left\langle\mathcal{H}^{\left(p_{1}\right)}\left(\frac{Y-Y^{\prime}}{\sqrt{\gamma}}, s\left(1-\frac{\theta_{1}}{p_{1}^{2}}\right)\right), \kappa^{(0,0)}+\frac{Y}{\sqrt{p}}\right\rangle \mathcal{H}^{\left(p_{2}\right)}\left(\frac{Y^{\prime}}{\sqrt{1-\gamma}}, s\left(1-\frac{\theta_{2}}{p_{2}^{2}}\right)\right) d^{n} Y^{\prime} . \\
& \sum_{p_{1}+p_{2}=p+1}^{p_{1}, p_{2}>\sqrt{p}} \int_{0}^{p_{1}^{2}} d \theta_{1} \int_{0}^{p_{2}^{2}} d \theta_{2} \cdot \gamma^{-n / 2}(1-\gamma)^{-n / 2} \cdot \frac{1}{p+1} \cdot e^{-\frac{\left|Y-Y^{\prime}\right|^{2}}{2 \gamma}-\frac{\mid Y^{\prime 2}}{2(1-\gamma)} .} \\
& \left.\left\langle\mathcal{H}^{\left(p_{1}\right)}\left(\frac{Y-Y^{\prime}}{\sqrt{\gamma}}, s\right)\right), \kappa^{(0,0)}+\frac{Y}{\sqrt{p}}\right\rangle \mathcal{H}^{\left(p_{2}\right)}\left(\frac{Y^{\prime}}{\sqrt{1-\gamma}}, s\right) d^{n} Y^{\prime} .
\end{aligned}
$$

We did not include the factor $\Lambda^{p} \cdot p^{\frac{4-n}{2}}$ because it is a part of the inductive assumption. This remainder is estimated in the following way.

First we consider

$$
R_{1}=\left(\left|k^{(0)}+\frac{Y-Y^{\prime}}{\sqrt{p} \gamma}\right|^{2}-1\right)+\left(\left|k^{(0)}+\frac{Y^{\prime}}{\sqrt{p}(1-\gamma)}\right|^{2}-1\right)
$$

As before, consider the domain where

$$
\left|Y-Y^{\prime}\right| \leq D_{9} \sqrt{\ln \left(p k^{(0)}\right)},\left|Y^{\prime}\right| \leq D_{10} \sqrt{\ln \left(p k^{(0)}\right)} .
$$

We write

$$
R_{1}=\frac{\left|Y-Y^{\prime}\right|^{2}}{p \cdot \gamma^{2}}+\frac{\left|Y^{\prime}\right|^{2}}{p \cdot(1-\gamma)^{2}}+D_{11}\left(\frac{\left|Y-Y^{\prime}\right|}{\sqrt{p} \gamma}+\frac{\left|Y^{\prime}\right|}{\sqrt{p}(1-\gamma)}\right) .
$$

In the Domain $A$

$$
\left|R_{1}\right| \leq \frac{D_{12} \ln \left(p k^{(0)}\right)}{p k^{(0)}}
$$

Therefore

$$
\begin{aligned}
& R_{2}=\exp \left\{-\theta_{1}\left|k^{(0)}+\frac{Y-Y^{\prime}}{\sqrt{p} \gamma}\right|^{2}-\theta_{2}\left|k^{(0)}+\frac{Y^{\prime}}{\sqrt{p} \gamma^{2}}\right|^{2}\right\} \\
& -\exp \left\{-\theta_{1}\right\} \cdot \exp \left\{-\theta_{2}\right\} \\
& \left.=\exp \left\{-\left(\theta_{1}+\theta_{2}\right)\right)\right\} \cdot\left[\operatorname { e x p } \left\{-\theta_{1}\left(\left|k^{(0)}+\frac{Y-Y^{\prime}}{\sqrt{p} \gamma}\right|^{2}-1\right)\right.\right.
\end{aligned}
$$




$$
\left.\cdot \exp \left\{-\theta_{2}\left(\left|k^{(0)}+\frac{Y^{\prime}}{\sqrt{p}(1-\gamma)}\right|^{2}-1\right)\right\}-1\right]
$$

and in the Domain A

$$
\left|R_{2}\right| \leq \exp \left\{-\left(\theta_{1}+\theta_{2}\right)\right\}\left(\frac{\theta_{1} \cdot D_{13}}{\sqrt{p} \gamma}+\frac{\theta_{2} \ln p}{\sqrt{p}(1-\gamma)}\right) .
$$

This shows that in the Domain A we can replace the exponent

$$
\exp \left\{-\theta_{1}\left|k^{(0)}+\frac{Y-Y^{\prime}}{\sqrt{p} \gamma}\right|^{2}-\theta_{2}\left|k^{(0)}+\frac{Y^{\prime}}{\sqrt{p}(1-\gamma)}\right|^{2}\right\}
$$

by $\exp \left\{-\left(\theta_{1}+\theta_{2}\right)\left(k^{(0)}\right)^{2}\right\}$ and the remainder will be not more than $\frac{D_{14} \ln p}{\sqrt{p}}$. This is enough for our purposes. In the Domain B the estimates are similar because again the main contribution to the integral comes from $\left|Y-Y^{\prime}\right| \leq D_{9} \sqrt{\ln p},\left|Y^{\prime}\right| \leq D_{10} \sqrt{\ln p}$. In other words, in the Domain B we can replace the product of the Gaussian factors and $\mathcal{H}^{(p)}$ by

$$
\exp \left\{-\frac{1}{2}\left|Y-Y^{\prime}\right|^{2}-\frac{1}{2}\left|Y^{\prime}\right|^{2}\right\} \text {. }
$$

This is also enough for our purposes.

The next remainder of Type I comes from the difference between the sum over $\gamma$ and the corresponding integral. The remainder $\beta_{p}^{(3)}(Y, s)$ is the difference between the sum and the corresponding integral over $\gamma$ from 0 to 1 . It is easy to check that this difference is not more than $\frac{D_{14}}{\sqrt{p}}$.

5.2. The Remainders of Type II and III. All remainders of Type II appear because we use the sums (over $p_{1}$ ) instead of the integrals. We use a linear interpolation to define $\delta(\gamma, Y, s)$ for all $\gamma$. From our inductive assumptions it follows that $\left|\delta_{p}(\gamma, Y, s)\right| \leq \frac{D_{16}}{\sqrt{p}}$. Therefore, the remainders which follow from the difference between the sum and the integral also satisfy this estimate.

It remains to consider quadratic expressions of $\delta_{p}(\gamma, Y, s)$. The Gaussian density is present in all these expressions. Therefore, all the remainders are not more than $\frac{D_{17}}{p}$.

\section{Final Steps in the Proof of the Main Result. The Formulation of the Main Theorem}

In this section we consider our procedure for $p>p_{0}=N$. Introduce the sequence $p_{m}, p_{m}=(1+\epsilon) p_{m-1}=(1+\epsilon)^{m} p_{0}, m>0$, where $\epsilon>0$ is small (see below). These are the values of $p$ when we make the change of parameters, i.e. rescaling. For $p \neq p_{m}$, no changes are done.

In $\S 4$ the choice of the fixed point $H^{(0)}$ was explained and the corresponding functions $\Phi_{j}^{(u)}, \Phi_{j^{\prime}}^{(n)}$ were introduced. Also we have the 
stable subspace of our linearized map. Consider $p, p_{m}<p<p_{m+1}$, $m \geq 0$. By induction, assume that we have an interval on the time axis $S^{(m)}=\left[S_{-}^{(m)}, S_{+}^{(m)}\right]$ such that for all $s \in S^{(m)}, r<p$, we have the representation (see also (24) for the definition of $\Lambda(s)$ ):

$$
\begin{array}{r}
\tilde{g}_{r}(Y, s)=Z \cdot \Lambda^{r}(s) \cdot r^{\frac{4-n}{2}} \cdot\left(H^{(0)}(Y)+\delta^{(r)}(Y, s)\right) \cdot \frac{1}{(2 \pi)^{n / 2}} e^{-\frac{|Y|^{2}}{2}}, \\
r<p .
\end{array}
$$

If $\gamma=\frac{r}{p-1}$, then

$$
\begin{aligned}
\delta^{(r)}(Y, s)= & \sum_{j=1}^{l^{(u)}}\left(b_{j, p-1}^{(u, m)}+\beta_{j, r}^{(u)}\right) \gamma^{\alpha_{j}^{(u)}} \Phi_{j}^{(u)}(Y) \\
& +\sum_{j^{\prime}=1}^{l^{(n)}}\left(b_{j^{\prime}, p-1}^{(n, m)}+\beta_{j^{\prime}, r}^{(n)}\right) \Phi_{j}^{(n)}(Y)+\Phi^{(s t)}(Y, \gamma) .
\end{aligned}
$$

Remark that in this expression $b_{j, p-1}^{(u, m)}, b_{j^{\prime}, p-1}^{(n, m)}$ do not depend on $r$. The terms $\beta_{j, r}^{(u)}, \beta_{j^{\prime}, r}^{(n)}$ are small corrections to the main terms $b_{j, p-1}^{(u, m)}, b_{j^{\prime}, p}^{(n, m)}$ and are also functions of $b_{j}^{(u, m)}, b_{j^{\prime}}^{(n, m)}$. Our next inductive assumption says that $b_{j, p_{m}}^{(u, m)}, b_{j^{\prime}, p_{m}}^{(n, m)}$ satisfy the inequalities

$$
-B_{1} \rho_{1}^{m} \leq b_{j, p_{m}}^{(u, m)}, b_{j^{\prime}, p_{m}}^{(n, m)} \leq B_{1} \rho_{1}^{m}
$$

where $B_{1}$ is a positive constant. We can take $B_{1}=2$. The inductive assumption concerning the corrections $\beta_{j, r}^{(u)}, \beta_{j^{\prime}, r}^{(n)}$ says that

$$
\begin{gathered}
\left|\beta_{j, r}^{(u)}\right|,\left|\beta_{j^{\prime}, r}^{(n)}\right| \leq B_{2} \rho_{2}^{m} \quad \text { for all } 1 \leq j \leq l^{(u)}, 1 \leq j^{\prime} \leq l^{(n)}, \\
\left|\frac{\partial \beta_{j, r}^{(u)}}{\partial b_{j_{1}, r}^{(u, m)}}\right|,\left|\frac{\partial \beta_{j, r}^{(u)}}{\partial b_{j_{1}^{\prime}, r}^{(n, m)}}\right|,\left|\frac{\partial \beta_{j^{\prime}, r}^{(n)}}{\partial b_{j_{1}, r}^{(u, m)}}\right|\left|\frac{\partial \beta_{j^{\prime}, r}^{(n)}}{\partial b_{j_{1}^{\prime}, r}^{(n, m)}}\right| \leq B_{3} \cdot \rho_{2}^{m}
\end{gathered}
$$

Here $0<\rho_{2}<\rho_{1}$ and $B_{2}$ are other constants.

The function $\Phi^{(s t)}(Y, \gamma)$ belongs to the stable subspace of the linearized semi-group (see $\S 3$ ) and satisfies the inequality:

$$
\left\|\Phi^{(s t)}(Y, \gamma)\right\|_{X} \leq B_{3} \rho_{3}^{m}
$$

where $B_{3}>0,0<\rho_{3}<1$ are constants. At one step of our procedure $p-1$ is replaced by $p, \gamma$ is replaced by $\gamma^{\prime}=\gamma \cdot \frac{p-1}{p}$ and $\gamma^{\alpha_{j}^{(u)}}$ is replaced by $\left(1+\frac{1}{p-1}\right)^{\alpha_{j}^{(u)}} \cdot\left(\gamma^{\prime}\right)^{\alpha_{j}^{(u)}}, b_{j}^{(u, m)}+\beta_{j, r}^{(u)}$ is replaced by $\left(b_{j}^{(u, m)}+\beta_{j, r}^{(u)}\right)\left(1+\frac{1}{p-1}\right)^{\alpha_{j}^{(u)}}$. At the end of the interval $p_{m}<p<p_{m+1}$ 
the variable $b_{j, p_{m}}^{(u, m)}$ acquires the factor

$$
\prod_{p_{m}<p<p_{m+1}}\left(1+\frac{1}{p-1}\right)^{\alpha_{j}^{(u)}} \approx(1+\epsilon)^{\alpha_{j}^{(u)} .}
$$

For the neutral part of the spectrum the variable $b_{j^{\prime}, p_{m}}^{(n, m)}$ remains the same because $\alpha_{j^{\prime}}^{(n)}=0$. The stable part is contracting.

Now we discuss $\delta^{(p)}(Y, 1)$ using $(7)$. As in $\S 4, \delta^{(p)}(Y, 1)$ consists of three parts.

Part I. In all $\delta^{(r)}, r<p$, the main term is the one which contains the basic parameters $b_{j, p}^{(u, m)}, b_{j^{\prime}, p}^{(n, m)}$. Consider the terms in (7) which are linear in $b_{j, p-1}^{(u)}, b_{j^{\prime}, p-1}^{(n)}$. As it follows from the definition of the linearized group and its spectrum, for unstable eigen-vectors we get the factor $\left(1+\frac{1}{p-1}\right)^{\alpha_{j}^{(u)}} b_{j, p-1}^{(u, m)}$. For the neutral part we get the factor 1 . We put $b_{j, p}^{(u, m)}=b_{j, p-1}^{(u, m)}$. $\left(1+\frac{1}{p}\right)^{\alpha_{j}^{(u)}}, b_{j^{\prime}, p}^{(n, m)}=b_{j^{\prime}, p-1}^{(n)}$. The vector corresponding to the stable subspace is transformed accordingly.

Part II. All remainders which arise because the formulas for finite $p$ are different from the limiting formulas. These remainders were discussed in $\S 4$. The result is written as a linear combination of $\Phi_{j}^{(u)}, \Phi_{j^{\prime}}^{(n)}$ and a vector from the stable subspace. The corresponding terms are included in $\beta_{j, p}^{(u)}, \beta_{j^{\prime}, p}^{(n)}$ and the function from the stable subspace.

Part III. The term which is the sum of all quadratic functions of all $\delta^{(r)}$. Again we expand it using the functions $\Phi_{j}^{(u)}, \Phi_{j^{\prime}}^{(n)}$ and the stable subspace. The result is included in $\beta_{j, p}^{(u, m)}, \beta_{j^{\prime}, p}^{(n, m)}$ and $\Phi_{p}^{(s t)}(Y)$ from the stable subspace.

Finally we have

$$
b_{j, p}^{(u, m)}=b_{j, p-1}^{(u, m)}\left(1+\frac{1}{p}\right)^{\alpha_{j}^{(u)}}, \quad b_{j, p}^{(n, m)}=b_{j, p-1}^{(n, m)}
$$

and the formulas for $\beta_{j, p}^{(u, m)}, \beta_{j^{\prime}, p}^{(n, m)}$ and $\Phi_{p}^{(s t)}(Y)$.

This procedure is used until $p<p_{m+1}$. When $p=p_{m+1}$, then in addition we make rescaling and introduce new variables

$$
\begin{gathered}
b_{j, p_{m+1}}^{(u, m+1)}=b_{j, p_{m+1}}^{(u, m)}+\beta_{j, p_{m+1}}^{(u, m)}, \\
b_{j^{\prime}, p_{m+1}}^{(n, m+1)}=b_{j^{\prime}, p_{m+1}}^{(n, m)}+\beta_{j^{\prime}, p_{m+1}}^{(n, m)} .
\end{gathered}
$$




$$
\begin{aligned}
& \text { Let } \Delta_{m+1}^{(m+1)}=\left[-B_{1} \rho_{1}^{m+1}, B_{1} \rho_{1}^{m+1}\right] \text { and } \\
& \Delta_{m}^{(m+1)}=\left\{\left(b_{j, p_{m}}^{(u, m)}, b_{j^{\prime}, p_{m}}^{(n, m)}\right):-B_{1} \rho_{1}^{m+1} \leq b_{j, p_{m+1}}^{(u)}, b_{j^{\prime}, p_{m+1}}^{(n)} \leq B_{1} \rho_{1}^{m+1}\right\} .
\end{aligned}
$$

It follows easily from the estimates of $\beta_{j, p_{m+1}}^{(u, m+1)}, \beta_{j^{\prime}, p_{m+1}}^{(n, m+1)}$ that $\Delta_{m}^{(m+1)} \subseteq$ $\Delta_{m}^{(m)}$. If

$$
\Delta_{0}^{(m)}=\left\{\left(b_{j}^{(u)}, b_{j^{\prime}}^{(n)}\right):\left(b_{j, m}^{(u, m)}, b_{j^{\prime}, m}^{(n, m)}\right) \in \Delta_{m}^{(m)}\right\},
$$

then $\Delta_{0}^{(m)}$ is a decreasing sequence of closed intervals. The intersection $\bigcap_{m} \Delta_{0}^{(m)}$ gives us the values of parameters for which $\delta^{(p)} \rightarrow 0$ as $p \rightarrow \infty$.

We make also some shortening of the time interval $S^{(m)}$. In the formulas for $\delta^{(r)}$ there are several remainders which appear because we replace in all expressions $s^{\prime}$ and $s^{\prime \prime}$ by $s$. We estimate these remainders using the fact that our functions satisfy the Lipschitz condition with respect to the time variable. The maxima of these functions decay as some power of $p$. We choose the interval $S^{(m+1)} \subset S^{(m)}$ so that for all $s \in S^{(m+1)}$ the basic inclusion $\Delta_{m}^{(m+1)} \subset \Delta_{m}^{(m)}$ remains valid. The difference $S^{(m)} \backslash S^{(m+1)}$ consists of two intervals whose lengths decay exponentially with $m$. Therefore $\bigcap_{m} S^{(m)}=\left[S_{-}, S_{+}\right]$is an interval of positive length.

The transformation $\left(b_{j, p_{m+1}}^{(u, m+1)}, b_{j^{\prime}, p_{m+1}}^{(n, m+1)}\right) \rightarrow\left(b_{j, p_{m}}^{(u, m)}, b_{j^{\prime}, p_{m}}^{(n, m)}\right)$ is given by smooth functions and is close to the identity map. The last step in the renormalization procedure is the replacement in all $\delta^{(r)}, r<p_{m+1}$ the variables $b_{j, p_{m}}^{(u, m)}, b_{j^{\prime}, p_{m}}^{(n, m)}$ by their expressions through $b_{j, p_{m+1}}^{(u, m+1)}, b_{j^{\prime}, p_{m}+1}^{(n, m+1)}$. The form of $\delta^{(r)}$ in new variables remains essentially the same.

\section{The Choice of Constants}

The main constants which are used in the construction are the following:

1. $k^{(0)}$ determines the center of the domain where $v(k, 0)$ is concentrated;

2. $D_{1}$ is the constant which determines the size of the neighborhood where $v(k, 0)$ is concentrated;

3. $\rho_{1}, B_{1}$ determine the size of the intervals where $b_{j}^{(u)}, b_{j^{\prime}}^{(n)}$ vary;

4. $\rho_{2}, B_{2}$ determine the upper bounds of the perturbations $\beta_{j, r}^{(u)}$, $\beta_{j^{\prime}, r}^{(n)}$;

5. $\lambda_{1}$ determines the power of decay of $g_{r}$ in the domain $B$.

6. $N$ is the number of steps where the procedure was done numerically;

7. $\epsilon$ determines the values of $p$ where the renormalization is done.

The value of $k^{(0)}$ should be sufficiently large. The constant $B_{1}$ should be small but $\rho_{1}<1$ should not be too small in order to make the corrections coming from the quadratic part of our formulas to be 
smaller than the main term in the linear part. Moreover, it cannot be too small in order that we could choose the next interval $\Delta^{(m+1)}$. The parameter $\lambda_{1}$ is a function of $D_{1}$. The value of $D_{1}$ determines the estimates in the domain $B$ which decay as $\frac{1}{\left(k^{(0)}\right)^{\lambda_{1}}}$. The value of $\epsilon$ is chosen so small that we can write with a good precision the action of the linearized renormalization group.

Now we formulate the main result of this paper.

TheOREM 1 (Main Theorem). Take a $\left(n^{2}+5 n-2\right) / 2$-parameter family of initial conditions described in $\S 4$ provided that all constants satisfy the needed inequalities. Then one can find an interval $S=\left[S_{-}, S_{+}\right]$, the functions $Z(s), \Lambda(s)$, and the values $b_{j}^{(u)}=b_{j}^{(u)}(s), b_{j^{\prime}}^{(n)}=b_{j^{\prime}}^{(n)}(s)$ of parameters so that $\left.a_{1}\right)$. For $|Y| \leq D_{1} \sqrt{p k^{(0)}}$,

$$
\begin{aligned}
\tilde{g}_{p}(Y, s) & =g_{p}\left(k^{(p)}+\sqrt{p k^{(0)}} Y, s\right) \\
& =Z \cdot \Lambda^{p}(s) \cdot p^{\frac{4-n}{2}} \cdot\left(H^{(0)}(Y)+\delta^{(p)}(Y, s)\right) \cdot \frac{1}{(2 \pi)^{n / 2}} e^{-\frac{|Y|^{2}}{2}}
\end{aligned}
$$

and $\sup _{Y}\left|\delta^{(p)}(Y, s)\right| \rightarrow 0$ as $p \rightarrow \infty$. Here $H^{(0)}(Y)$ is the fixed point of our renormalization group for which $x_{1}=\cdots=x_{n}=2 / n$.

$\left.a_{2}\right)$. For $|Y|>D_{1} \sqrt{p k^{(0)}}$,

$$
\left|\tilde{g}_{p}(Y, s)\right| \leq \frac{B_{1} \Lambda_{p}(s)}{p^{\lambda_{1}}}
$$

The function $\Lambda(s)$ is strictly increasing on $S$. Moreover, for $s \in S$, we have

$$
\Lambda^{\prime}(s) \geq B>0
$$

where $B>0$ is another constant independent of $s$.

\section{Critical Value of Parameters and Behavior of Solutions near the Singularity Point}

We return back to the first formulas:

$$
\begin{aligned}
v_{A}(k, t)= & \exp \left\{-t|k|^{2}\right\} A \cdot v(k, 0) \\
& +\int_{0}^{t} \exp \left\{-(t-s)|k|^{2}\right\} \cdot \sum_{p>1} A^{p} g_{p}(k, s) d s .
\end{aligned}
$$

Take $t \in\left[S_{-}, S_{+}\right]$and find the values of parameters $b_{j}^{(u)}, b_{j^{\prime}}^{(n)}$ for which the main theorem holds. Put $A_{c r}(t)=\Lambda^{-1}(t)$. If so then $A^{p} g_{p}(k, t)$ is concentrated in the domain with the center at $\kappa^{(0)} p$ having the size 
$O(\sqrt{p})$ and there it takes values $O(p)$. This immediately implies that at $t$ the energy is infinite.

Consider $t^{\prime}<t$ and denote $\Delta t=t-t^{\prime}$. It follows from the properties of $\Lambda(s)$ (see the formulation of the main theorem) that $\Lambda\left(t^{\prime}\right) / \Lambda(t)=$ $(1-B \Delta t+O(\Delta t))$ for some constant $B>0$. Since $A_{c r}^{p} \cdot\left(\Lambda\left(t^{\prime}\right)\right)^{p}=$ $A_{c r}^{p} \cdot(\Lambda(t))^{p} \cdot\left(\Lambda\left(t^{\prime}\right) / \Lambda(t)\right)^{p}=(1-B \Delta t+o(\Delta t))^{p}$, it is clear that the terms in (26) with $p \leq O\left(\frac{1}{\Delta t}\right)$ are close to the limiting terms corresponding to $t$. For $p \gg O\left(\frac{1}{\Delta t}\right)$ the product $A_{c r}^{p}\left(\Lambda\left(t^{\prime}\right)\right)^{p}$ tends exponentially to zero and dominates other terms in the expression for $g_{p}$. Therefore for $t^{\prime}<t$ both the energy and the enstrophy are finite.

In the domain $|k| \leq O\left(\frac{1}{\Delta t}\right)$, the solutions grow as $|k|^{\frac{5-n}{2}}$. The extra factor $|k|^{\frac{1}{2}}$ appears because for any $k$ the values of $p$ for which the terms in (26) giving the essential contribution to the solution belongs to an interval of the size $O(\sqrt{|k|})=O(\sqrt{p})$. From this argument one can easily derive that $E\left(t^{\prime}\right)=\frac{O(1)}{(\Delta t)^{5}}$ and $\Omega\left(t^{\prime}\right)=\frac{O(1)}{(\Delta t)^{7}}$.

It is interesting to understand the form of the solution at $t=t_{c r}$ in the $x$-space. Some information can be obtained using (26). Consider the series $g\left(k, t_{c r}\right)=\sum_{p>1} A_{c r}^{p} g_{p}\left(k, t_{c r}\right)$. We neglect all remainders $\delta$ and this is reasonable. Then from our main theorem

$$
\tilde{g}_{r}\left(Y, t_{c r}\right)=Z\left(t_{c r}\right) \Lambda^{r}\left(t_{c r}\right) r^{\frac{4-n}{2}} \frac{1}{(2 \pi)^{n / 2}} e^{-\frac{|Y|^{2}}{2}} v,
$$

where $v=2 / n(1, \ldots, 1) \in R^{n}$ corresponds to our fixed point for which $x_{1}=\cdots=x_{n}=2 / n$. In this way since $A_{c r}=\Lambda\left(t_{c r}\right)^{-1}$ we have

$$
\sum_{p>1} A_{c r}^{p} g_{p}\left(k, t_{c r}\right)=Z\left(t_{c r}\right) \sum_{p>1} p^{\frac{4-n}{2}} \frac{1}{(2 \pi)^{n / 2}} \exp \left\{-\frac{1}{2}\left|\frac{k-k^{(p)}}{\sqrt{p k^{(0)}}}\right|^{2}\right\} v \text {. }
$$

Therefore the Fourier transform of $g\left(k, t_{c r}\right)$ has the form

$$
\begin{aligned}
\hat{g}\left(x, t_{c r}\right) & =\int_{R^{n}} e^{i\langle x, k\rangle} g\left(k, t_{c r}\right) d k \\
& =Z\left(t_{c r}\right) \sum_{p>1} p^{2} \cdot \frac{1}{\left(k^{(0)}\right)^{n / 2}} e^{i k^{(p)} \cdot x} \cdot e^{-\frac{1}{2} p k^{(0)}|x|^{2}} v .
\end{aligned}
$$

This expression shows that for all $x \neq 0$ the function $\hat{g}\left(x, t_{c r}\right)$ is finite but $\hat{g}\left(x, t_{c r}\right)$ tends to infinity as $O\left(\frac{1}{|x|^{6}}\right)$ as $x \rightarrow 0$. In this sense our solution is a tornado-like solution.

\section{Appendix I:}

\section{Hermite Polynomials and Their Basic Properties}

Take $\sigma>0$ and write

$$
H e_{n}^{(\sigma)}(x)=(-1)^{n} e^{\frac{\sigma x^{2}}{2}} \frac{d^{n}}{d x^{n}} e^{-\frac{\sigma x^{2}}{2}}, \quad n \geq 0 .
$$


It is clear that $H e_{n}^{(\sigma)}(x)=\sigma^{n} x^{n}+\cdots$, where dots mean terms of smaller degree. We shall call $H e_{n}^{(\sigma)}$ the $\mathrm{n}$-th Hermite polynomial. It is clear that $H e_{0}^{(\sigma)}(x)=1, H e_{1}^{(\sigma)}(x)=\sigma x, H e_{2}^{(\sigma)}(x)=\sigma^{2} x^{2}-\sigma$ and so on. In general, $H e_{n}^{(\sigma)}(x)=\sigma^{\frac{n}{2}} H e_{n}^{(1)}(\sqrt{\sigma} x)$. It is easy to check that

$$
\sigma x H e_{n}^{(\sigma)}(x)=H e_{n+1}^{(\sigma)}(x)+\sigma n H e_{n-1}^{(\sigma)}(x) .
$$

The Fourier transform of $H e_{m}^{(\sigma)}(x) e^{-\frac{\sigma x^{2}}{2}} \sqrt{\frac{\sigma}{2 \pi}}$ is $(i \lambda)^{m} e^{-\frac{\lambda^{2}}{2 \lambda}}$. This implies the formula for the convolution:

$$
\begin{aligned}
& \int_{\mathbb{R}^{1}} H e_{m_{1}}^{(\sigma)}(x-y) e^{-\frac{\sigma(x-y)^{2}}{2}} \sqrt{\frac{\sigma}{2 \pi}} \cdot H e_{m_{2}}^{(\sigma)}(y) e^{-\frac{\sigma y^{2}}{2}} \sqrt{\frac{\sigma}{2 \pi}} d y \\
& =H e_{m_{1}+m_{2}}^{(\sigma)}(x) e^{-\frac{\sigma x^{2}}{2}} \sqrt{\frac{\sigma}{2 \pi}} .
\end{aligned}
$$

Take positive $\gamma_{1}, \gamma_{2}, \gamma_{1}+\gamma_{2}=1$ and consider the convolution of $H e_{m_{1}}^{(\sigma)}\left(\frac{x}{\sqrt{\gamma_{1}}}\right) e^{-\frac{\sigma x^{2}}{2 \gamma_{1}}} \cdot \sqrt{\frac{\sigma}{2 \pi \gamma_{1}}}$ and $H e_{m_{2}}^{(\sigma)}\left(\frac{x}{\sqrt{\gamma_{2}}}\right) e^{-\frac{\sigma x^{2}}{2 \gamma_{2}}} \cdot \sqrt{\frac{\sigma}{2 \pi \gamma_{2}}}$. Their Fourier transforms are $\left(i \lambda \sqrt{\gamma_{1}}\right)^{m_{1}} e^{-\frac{\lambda^{2} \gamma_{1}}{2 \sigma}}$ and $\left(i \lambda \sqrt{\gamma_{2}}\right)^{m_{2}} e^{-\frac{\lambda^{2} \gamma_{2}}{2 \sigma}}$ respectively. The product of these two functions is $\gamma_{1}^{\frac{m_{1}}{2}} \gamma_{2}^{\frac{m_{2}}{2}}(i \lambda)^{m_{1}+m_{2}} e^{-\frac{\lambda^{2}}{2 \sigma}}$. Therefore the convolution is $\gamma_{1}^{\frac{m_{1}}{2}} \cdot \gamma_{2}^{\frac{m_{2}}{2}} H e_{m_{1}+m_{2}}^{(\sigma)}(x) e^{-\frac{\sigma x^{2}}{2}}$.

\section{References}

[C] M. Cannone, Harmonic analysis tools for solving the incompressile NavierStokes equations, Handbook of Mathematical Fluid Dynamics, vol. 3, 2002.

[Cl] Clay Mathematical Institute, The millennium prize problems, 2006.

[F-T] C. Foias and R. Temam, Gevrey classes of regularity for the solutions of the Navier-Stokes equations, J. of Funct. Anal. 87 (1989) 359-369.

[GM] Y. Giga, T. Miyakawa, Navier-Stokes flow in $R^{3}$ with measures as initial vorticity and Morrey spaces, Commu. Partial Differential Equations, 14 (1989) 577-618.

[H] E. Hopf, Über die Anfanswertaufgabe für die hydrodynamischen Grundgleichungen, Math. Nachr, 4 (1951) 213-231.

[HC] E. Hopf, The partial differential equation $u_{t}+u u_{x}=\mu u_{x x}$, Comm. Pure Appl. Math., 3 (1950) 201-230.

J. Cole, On a quasilinear parabolic equation occurring in aerodynamics, Quart. Appl. Math., 9 (1951) 225-236.

[K] T. Kato, Strong $L^{p}$-solution of the Navier-Stokes equation in $R^{m}$, with applications to weak Solutions, Math. Zeitschrift 187 (1984) 471-480.

[La] O. Ladyzenskaya, The mathematical theory of viscous incompressible flow, New York, Gordon and Breach Science Publishers, 1969.

[Le] J. Leray, Étude de diverses équations intégrales non linéaires et de quelques problémes que pose l'hydrodynamique, J. Math. Pures Appl. 12 (1993) 1-82.

[Li] D. Li, On a nonlinear recurrent relation, to appear in J. Stat. Phy.

[LS] D. Li and Ya.G. Sinai, Blow Ups of Complex Solutions of the 3D-Navier-Stokes System, J. of Eur. Math. Soc., to appear.

[PS] P. Polacik and V. Sverak, Zeros of complex caloric functions and singularities of complex viscous Burgers equation, ARXIV:0612506. 
[Si 1] Ya.G. Sinai, Power series for solutions of the Navier-Stokes system on $R^{3}$, Journal of Stat. Physics 121(516) (2005) 779-804.

[Si 2] Ya.G. Sinai, Diagrammatic approach to the 3D-Navier-Stokes system, Russian Math. Surveys 60(5) (2005) 47-70.

[Si 3] Ya.G. Sinai, Separating solution of a recurrent equation, Regular and Chaotic Dynamics 12(5) (2007) 490-501.

[Y] V.I. Yudovich, The linearization method in hydrodynamical stability theory, Trans. Math. Mon. Amer. Math. Soc., Providence, RI, 74 (1984).

School of Mathematics, Institute for Advanced Study, Princeton, NJ 08540

E-mail address: dongli@math.ias.edu

Mathematics Department, Princeton University, Princeton, NJ 08540

AND

Landau Institute of Theoretical Physics, Moscow, Russia

E-mail address: sinai@math.princeton.edu 